\title{
Antigen footprint governs activation of the B cell receptor
}

\author{
Alexey Ferapontov \\ Aarhus University \\ Marjan Omer \\ Aarhus University \\ Isabelle Baudrexel \\ Max Planck Institute https://orcid.org/0000-0002-3422-8340 \\ Jesper Nielsen \\ Aarhus University \\ Daniel Dupont \\ Aarhus University \\ Kristian Juul-Madsen \\ Aarhus University \\ Philipp Steen
}

Max Planck Institute of Biochemistry https://orcid.org/0000-0002-1454-9390

Steffen Thiel

Aarhus University

Thomas Vorup-Jensen

Aarhus University https://orcid.org/0000-0002-4140-6563

Ralf Jungmann

Max Planck Institute of Biochemistry https://orcid.org/0000-0003-4607-3312

Jørgen Kjems

Aarhus University

Søren Degn ( $\sim$ sdegn@biomed.au.dk)

Aarhus University https://orcid.org/0000-0001-5409-045X

\section{Article}

Keywords: B cell, B cell receptor, signaling, antigen, antibody, hapten, nanoscaffold, DNA PAINT, superresolution microscopy, activation mechanism

Posted Date: February 1st, 2022

DOI: https://doi.org/10.21203/rs.3.rs-1260549/v1 
License: (c) (i) This work is licensed under a Creative Commons Attribution 4.0 International License. Read Full License 


\section{Antigen footprint governs activation of the $B$ cell receptor}

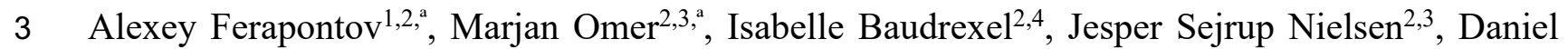

4 Miotto Dupont ${ }^{2,3}$, Kristian Juul-Madsen ${ }^{1}$, Philipp Steen ${ }^{4,5}$, Steffen Thiel ${ }^{1,2}$, Thomas Vorup-

5 Jensen ${ }^{1}$, Ralf Jungmann ${ }^{2,4,5}$, Jørgen Kjems ${ }^{2,3}$, Søren Egedal Degn ${ }^{1,2, *}$

$6 \quad$ These authors contributed equally.

$7{ }^{1}$ Department of Biomedicine, Aarhus University, Høegh-Guldbergs Gade 10, 8000 Aarhus C,

8 Denmark.

$9 \quad{ }^{2}$ Center for Cellular Signal Patterns (CellPAT), Aarhus University, Gustav Wieds Vej 14, 8000 10 Aarhus $C$, Denmark.

11 3interdisciplinary Nanoscience Center (iNANO), Aarhus University, Gustav Wieds Vej 14, 8000

12 Aarhus C, Denmark.

$13{ }^{4}$ Max Planck Institute of Biochemistry, Am Klopferspitz 18, 82152 Martinsried, Germany.

$14{ }^{5}$ Faculty of Physics and Center for Nanoscience, Ludwig Maximilian University, Munich,

15 Geschwister-Scholl-Platz 1, 80539 Munich, Germany.

16

17 *Correspondence to S.E.D.sdegn@biomed.au.dk 
Antigen binding by $\mathrm{B}$ cell receptors (BCRs) on cognate B cells elicits a response that eventually leads to production of antibodies. However, it is unclear what the distribution of BCRs is on the naïve B cell and how antigen binding triggers the first step in BCR signaling. Using DNA-PAINT

22 super-resolution microscopy, we find that most BCRs are present as monomers, dimers, or lower23 order oligomers on resting B cells, with a nearest-neighbor inter-Fab distance of 20-30 nm. We 24 leverage a Holliday junction nanoscaffold to engineer monodisperse model antigens with 25 precision-controlled affinity and valency, and find that the antigen exerts agonistic effects on the 26 BCR as a function of increasing affinity and avidity. Monovalent macromolecular antigens can 27 activate the $\mathrm{BCR}$ at high concentrations, whereas micromolecular antigens cannot, demonstrating 28 that antigen binding does not directly drive activation. Based on this, we propose a novel BCR 29 activation model determined by the antigen footprint.

31 Keywords: B cell, B cell receptor, signaling, antigen, antibody, hapten, nanoscaffold, DNA32 PAINT, super-resolution microscopy, activation mechanism. 


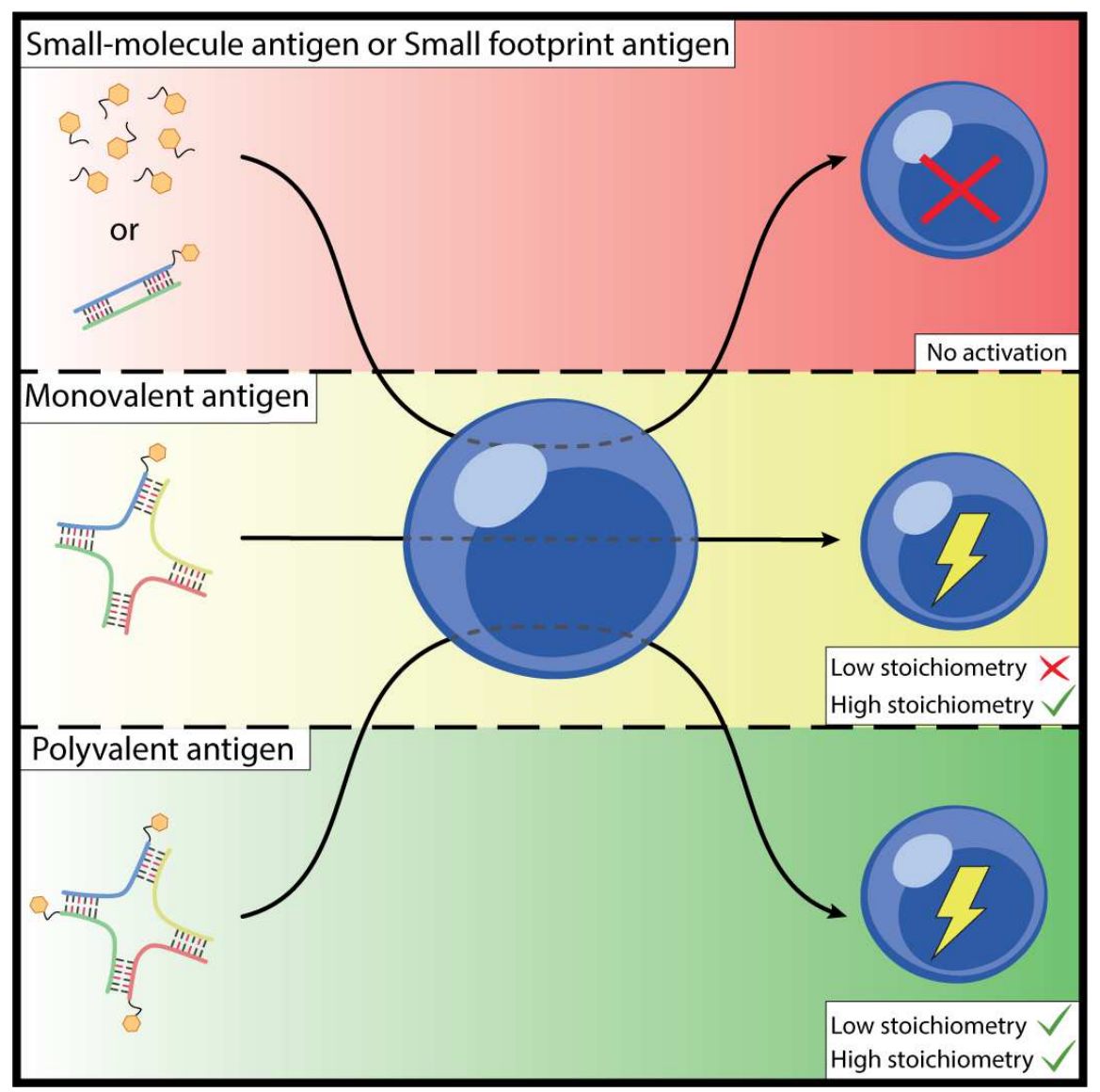




\section{Introduction}

The humoral branch of the adaptive immune system plays a central role in combating pathogens through the production of antibodies and immunological memory governed by B cells $\mathrm{s}^{1,2}$. B cells recognize antigens via their membrane-bound immunoglobulin (mIg), which, in conjunction with two transmembrane proteins $\operatorname{Ig} \alpha$ and $\operatorname{Ig} \beta$ (CD79A and CD79B, respectively), functions as a B cell receptor $(\mathrm{BCR})^{3,4}$. Antigen binding by the $\mathrm{BCR}$ triggers a signaling cascade that ultimately results in B cell proliferation and differentiation into plasma cells, germinal center and memory B cells. The discrete steps in the signaling cascade downstream of BCR activation are well-defined ${ }^{5}$, with the first critical step being phosphorylation of the CD79 ITAMs by the membrane-associated kinase Lyn and the cytosolic Src-family kinase Syk. Downstream signaling drives activation of the RAS/ERK, JNK and p38 pathways, while $\mathrm{IP}_{3}$ activation causes $\mathrm{Ca}^{2+}$ release and NFAT activation. Secondary to initial activation, cytoskeletal rearrangements drive a reorganization of BCRs on the surface, polarizing the cell, and forming a robust signaling complex. However, despite decades of intense scrutiny, it remains unclear exactly how the initial engagement of the BCR by antigen drives the earliest step in the activation of $\mathrm{B}$ cells ${ }^{6,7,8}$.

The classical view of receptor activation is that antigen-driven cross-linking of monomeric BCRs drives a localized concentration of ITAMs on the intracellular leaflet of the membrane, which enables scaffolding of kinases and adaptor proteins ${ }^{9,10}$. This model thus proposes that BCRs exist as monomers on the membrane of resting B cells. Evidence derived from Förster resonance energy transfer (FRET) and direct stochastic optical reconstruction microscopy (dSTORM) has suggested that BCRs spatially reorganize and form large clusters upon binding of multivalent antigens that can cross-link the BCRs to prompt receptor activation ${ }^{11,12,13}$.

Yet it has also been reported that monovalent antigen is able to activate the BCR, casting doubt upon the validity of the cross-linking model. Using B cells specific for a peptide derived from hen egg lysozyme, Kim et al. found that binding of the BCR by monovalent antigen, but not a Fab fragment targeting the $\mathrm{BCR}$, could induce receptor activation, although the monovalent antigen failed to promote subsequent antigen presentation ${ }^{14}$. Similar findings were presented for transnuclear B cells recognizing a peptide from ovalbumin ${ }^{15}$. Several studies have proposed that differential actions of Src family kinases and Syk could fine-tune the responsiveness of the BCR to monovalent versus multivalent ligands $s^{8,16,17}$. To rationalize such findings, an alternative model 
67 for BCR activation, the dissociation-activation model, proposed that under resting conditions

68 BCRs are found in microclusters with their Fab arms closely packed ${ }^{8}$. Ostensibly, in this resting configuration, the ITAMs of CD79 are packed so tightly that it precludes kinase access. The binding of antigen is envisioned to open the Fab arms, in turn dispersing the ITAMs to permit 71 kinase activity ${ }^{8}$.

It remains unclear, however, how BCRs would sense the binding of monovalent antigen, given the known flexibility of the hinge region across immunoglobulin isotypes used in BCRs and the lack of structural evidence for allosteric transmission through the heavy chain ${ }^{18}$. In line with this, other studies found no evidence for monovalent antigens as drivers of BCR activation ${ }^{19,20}$.

To date, most studies on soluble antigen interactions with the BCR have been based on the use of haptenized protein carriers or peptide antigens. Such antigen preparations have inherent issues precluding definitive control of valency. Direct haptenization of a carrier at any defined molar stoichiometry invariably yields an ensemble of valencies with a Poisson distribution around the defined stoichiometric center. A prevalent problem encountered with peptides is a tendency for subspecies aggregation, depending on hydrophobic patches and the experimental environment, such as $\mathrm{pH}$ and the presence of solutes and proteins ${ }^{21}$. Hence, it is very difficult to exclude that a minor fraction of monovalent antigen preparations in fact contains multivalent subspecies.

Here, we took an unbiased approach to address two fundamental questions to understand BCR activation, namely: 1) What is the resting state distribution of BCRs on the B cell surface, and 2) What are the minimal requirements for antigen-driven activation of the BCR?

We employed DNA points accumulation for imaging in nanoscale topography (DNA-PAINT) ${ }^{22}$ to resolve the resting distribution of BCRs on the cell surface, establishing that most BCRs are present as monomers, dimers, or lower-order oligomers on resting B cells, with a nearest-neighbor interFab distance of approximately 20-30 nm. We then leveraged a 4-way junction nanoscaffold composed of chemically modified RNA strands, forming a defined quaternary complex, which resembles the Holliday junction (HJ) that occurs naturally in connection with homologous recombination. The complex can be recapitulated in vitro using 4 complementary synthetic oligonucleotides, each of which can be conjugated to biomolecules of interest and purified to homogeneity prior to self-assembly. Incorporation of locked nucleic acids and 2'-OMe RNA nucleotides ensures high thermal stability and resistance to degradation in biofluids, enabling cell 
97 targeting ${ }^{23}$. Here, we employed the nanoscaffold to engineer monodisperse, precision-controlled 98 mono- and polyvalent antigens with known affinities, and probed the minimal structural 99 requirements for BCR activation. We compared this to micromolecular antigens, and found that 100 antigen binding in itself is insufficient to drive activation, rather, activation requires a minimal 101 antigen footprint. 


\section{Results}

\section{BCRs are distributed on the membrane of naïve, resting $B$ cells}

To get a more detailed understanding of the starting point for $\mathrm{B}$ cell activation, we investigated the BCR distribution on resting $\mathrm{B}$ cells using DNA-based point accumulation for imaging in nanoscale topography (DNA-PAINT) super-resolution microscopy. Imaging of the BCR was performed on untouched, naïve murine B lymphocytes, freshly isolated from B1-8hi knock-in mice ${ }^{24}$, and fixed in solution, leaving them unperturbed before preservation. Prior to permeabilization and staining, cells were centrifuged on glass channel slides, and both the IgM and IgD BCRs were quantitatively labeled with an anti-mouse-kappa-light chain nanobody ( $\kappa \mathrm{LC}-\mathrm{Nb})$, conjugated to a single docking strand $^{25}$ (Fig. 1A and Fig. S1). For image analysis, we selected either a rectangular or a round region of interest, covering the majority of the lymphocyte touching the surface (Fig. 1B). BCR clusters were identified by Density-Based Spatial Clustering of Applications with Noise (DBSCAN) which can detect clusters irrespective of their size without the pre-defined input of an estimated number of molecules ${ }^{26}$. In order to assess the number of individual BCRs for each BCR cluster, we made use of the programmable kinetics of DNA-PAINT and performed quantitative PAINT (qPAINT) analysis ${ }^{27}$ (Fig. S2). Based on the approximation that the influx rate of imager strands remains constant during the time of imaging, DNA-DNA binding kinetics serve as a direct read-out for the number of molecules present. After calibrating the influx rate with single binding sites (SBS), the cluster size in 31 cells was determined and clusters were grouped in monomers (1 molecule), dimers ( 2 molecules), oligomers (3-9 molecules) and islands ( $>9$ molecules) (Fig. 1C). We found that among all $\mathrm{BCR}$ molecules on the B cell surface, about $25 \%$ reside in monomers and $24 \%$ in dimers. Even though roughly $75 \%$ of all 'clusters' detected are monomers and dimers, the largest proportion of all BCRs on the cell surface resides in oligomeric arrangements of 3 9 molecules (37\%) (Fig. 1D). Larger islands $>9$ BCRs are rare and cannot be detected at all in 23\% of the cells, probably owing to their random orientation on the glass surface.

We analyzed the oligomers and islands further by determining the cluster density and found that most BCR clusters contain around one molecule per $1,000 \mathrm{~nm}^{2}$, irrespective of the molecule number per cluster (Fig. 1E). Assuming an even distribution of BCRs within clusters $>3$ molecules, this corresponds to an inter-Fab average distance of neighboring BCRs of about 20$30 \mathrm{~nm}$. These results make a direct BCR-BCR interaction in oligomers and islands unlikely, 
pointing towards external effects, like actin-confinement or membrane architecture, and not direct BCR-BCR-interaction, as the molecular mechanisms behind BCR assemblies. Our observations suggest that there is an equilibrium of isolated BCRs vs. those that are loosely associated as oligomers and islands.

In addition to cluster analysis, we estimated total BCR numbers on naïve murine B cells by extrapolating the measured BCR density to a spherical B cell surface ${ }^{28}$ and obtained an average of 10,000 BCRs per naïve B cell (Fig. 1F). However, because the labeling efficiency is likely below $100 \%$, we consider this value to be somewhat underestimated.

A

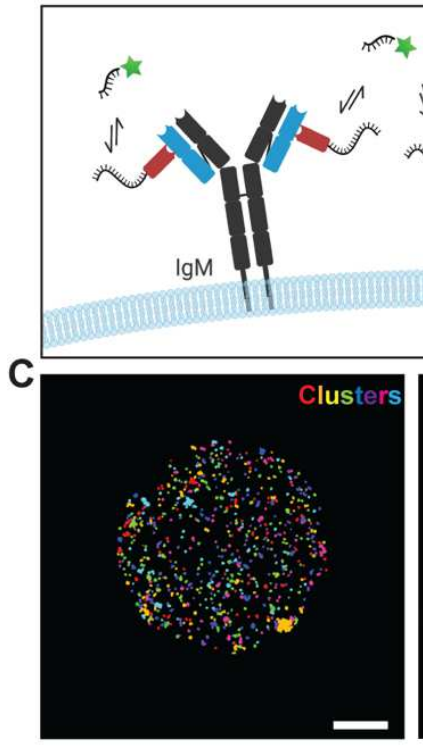

D

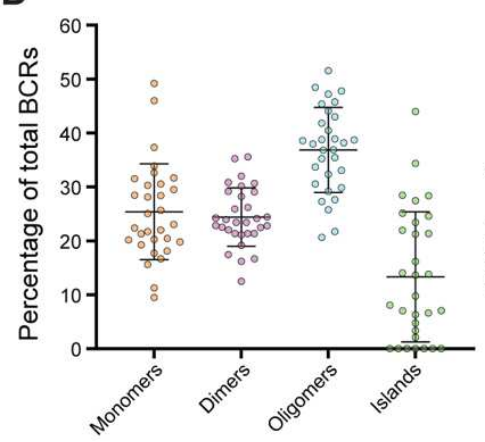

B
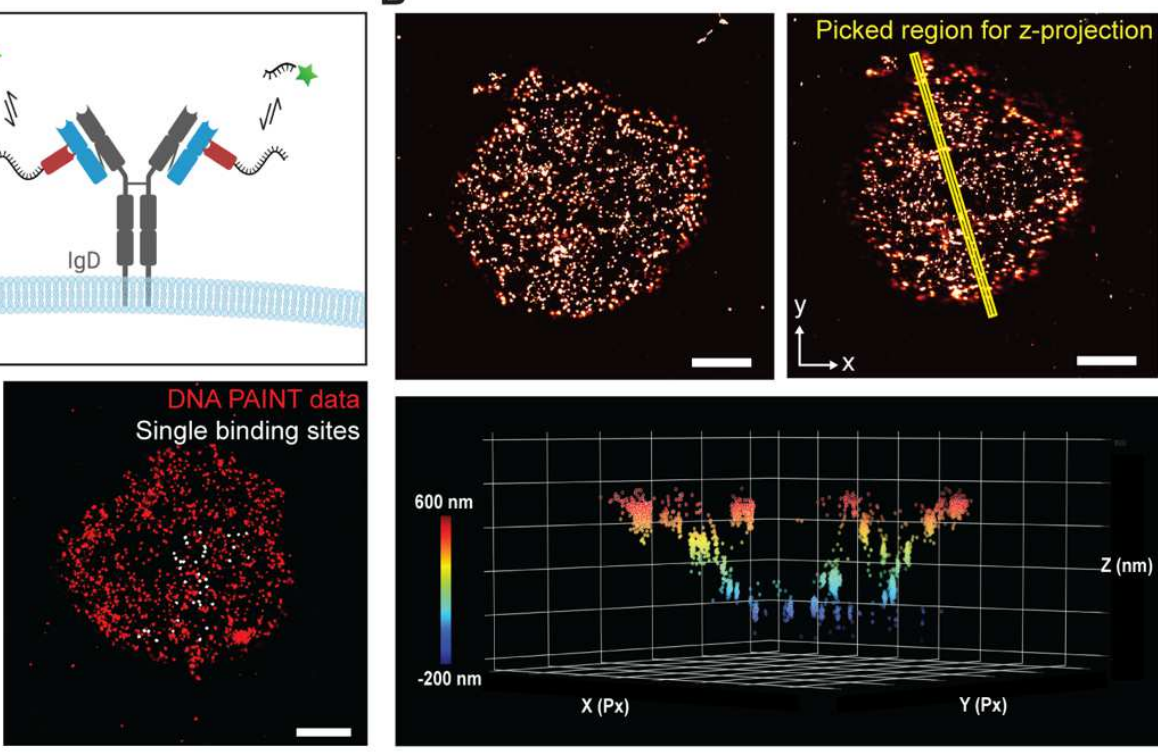

E

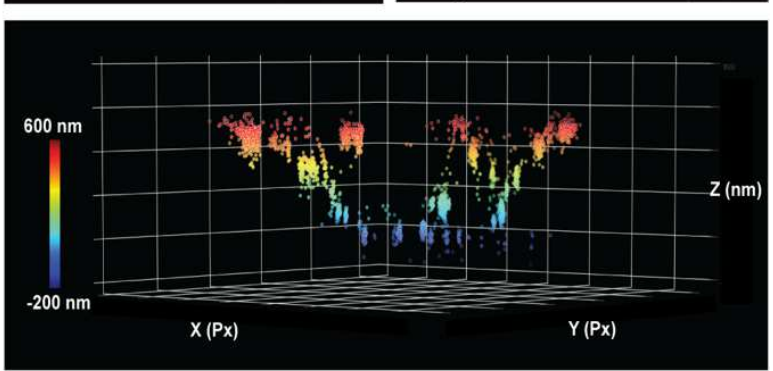

F
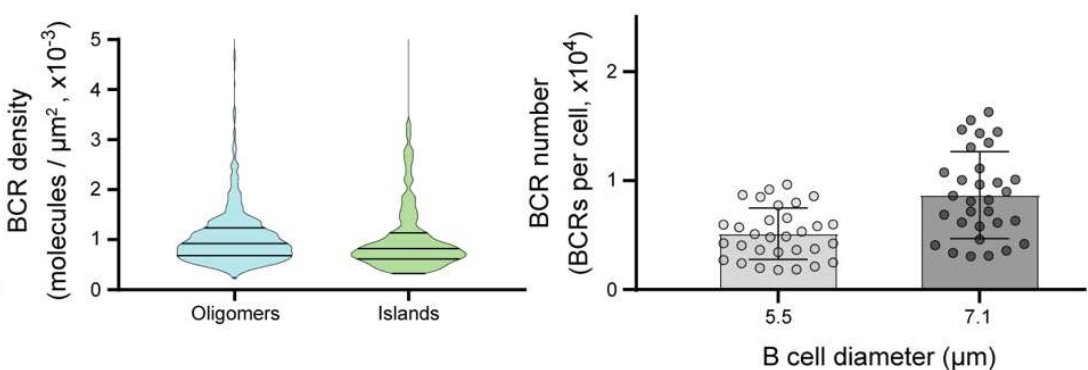

Figure 1. B cell receptor distribution of resting, naïve murine B cells. (A) An anti-mouse kappa light chain $\mathrm{Nb}$ which binds both IgM and IgD BCRs, conjugated site-specifically to a single docking strand, was used for imaging by the DNA-PAINT method (kappaLc: blue; Nb: red; DNA: black; Cy3b: green). (B) Both 2D (top left panel) and 3D 
(bottom panel) images of the BCRs were acquired. Plotting a rectangular region in xyz (top right panel) shows a preservation of the round architecture of B cells (bottom panel). Px, pixels. (C) To extract the information on the number of BCRs on the B cell surface along with the surface area, representative regions of the imaged B cells were analyzed by DBSCAN cluster analysis to identify clusters (left panel). For kinetic calibration, single binding sites were picked (right panel, and Fig. S9). (D) After calculating the number of BCRs per cluster detected by DBSCAN, the percentage of BCRs found as monomers (1 molecule), dimers (2 molecules), oligomers (3-9 molecules) and clusters ( $>9$ molecules) was determined. Bars indicate mean \pm SD. (E) qPAINT analysis was used to calculate the BCR density on the imaged surface. The lines in the violin plots indicate the quartiles and the median. (F) Total BCR numbers were estimated to be around 6,000 for $5.5 \mu \mathrm{m}$ B cell diameter (literature) and 10,000 for $7.1 \mu \mathrm{m}$ diameter (measured). Bars indicate mean \pm SD. For images, all scale bars are $1 \mu \mathrm{m}$.

\section{Nanoscaffolding of antigen allows affinity and avidity based control of antibody binding}

Having established that BCRs are distributed on the B cell surface in the naïve, resting state, we proceeded to the question of the minimal molecular requirements for antigen-driven B cell activation. Leveraging the locked-nucleic acid-based nanoscaffold based on the HJ structure, we precision-engineered monodisperse and stoichiometrically defined model antigens with exact antigen valency from 0-3 units. We employed the well-characterized B1-8 system, displaying specificity for the hapten 4-hydroxy-5-nitrophenylacetate (NP) and the heteroclitic hapten 4hydroxy-3-iodo-5-nitrophenylacetate (NIP) ${ }^{29}$. By combining this with the $\mathrm{B} 1-8 \mathrm{i}^{30}$ and $\mathrm{B} 1-8 \mathrm{hi}^{24}$ mouse models of intermediate and high affinity, respectively, we could control antigen affinity across more than 2 orders of magnitude ( $>400$-fold).

Three of the oligos were either left non-functionalized or coupled to NP or NIP, while the fourth oligo was coupled to Alexa Fluor 647 dye (AF647) for subsequent detection. To remove unreacted oligos, products were purified by reverse phase high-pressure liquid chromatography (RP-HPLC), then analyzed by denaturing PAGE and liquid chromatography-mass spectrometry (LC-MS) to verify the conjugation efficiency and purity of conjugates (Fig. 2A and Fig. S3). HJ constructs displaying 0-3 copies of haptens and an AF647 fluorochrome (Fig. 2B) were prepared by combining oligo conjugates in equimolar ratio in a one-pot self-assembly reaction. Analysis of the various HJ-NP or HJ-NIP combinations by electrophoretic mobility shift assay showed welldefined bands of fully assembled HJs with AF647 fluorochrome incorporation (Fig. 2B and C). The relatively small size of NP and NIP molecules precluded a noticeable shift of the assembled HJ-NP or HJ-NIP constructs compared to the naked HJ. 
178 We employed flow-induced dispersion analysis (FIDA) ${ }^{31,32,33}$ to validate the monodispersity of our hapten-modified HJ constructs (Fig. S4B). Following incubation in the presence of serum albumin or all serum proteins, the apparent hydrodynamic radii of HJ-NP or HJ-NIP variants were measured within the interval of $R_{h} \sim 1.9-2.6 \mathrm{~nm}$ (Fig. 2D). Only a minor increase in $R_{h}(\sim 0.6 \mathrm{~nm}$ and $0.3 \mathrm{~nm}$ for $\mathrm{HJ}-3 \mathrm{xNP}$ and $\mathrm{HJ}-3 \mathrm{xNIP}$, respectively) was observed, and only for HJs bearing 3 NP or 3 NIP in the presence of serum, presumably due to association with serum proteins. This demonstrated that our hapten-modified $\mathrm{HJ}$ constructs remained monodisperse under the respective conditions and did not form aggregates or oligomers. In addition, we used FIDA to investigate the in-solution complex formation dynamics of hapten-conjugated HJs at various B1-8i anti-hapten mAb concentrations (Fig. S4D). No supramolecular immune complex formation was observed, but we saw antibody association with haptenated HJs corresponding to hapten valency; e.g., as expected, HJ-3xNIP bound maximally three anti-NP antibodies. In addition, based on the FIDA analysis we calculated the $\mathrm{K}_{\mathrm{D}}$ for the interaction of anti-NP mAb with HJ-1xNIP to be $\sim 20 \mathrm{nM}$, in line with previously reported affinities ${ }^{34}$. This demonstrated that conjugation of the hapten to the HJ does not alter antibody-antigen binding.

To test the binding capacity of HJ constructs with NP or NIP haptens displayed in various valencies, we performed bio-layer interferometry (BLI) assays on a label-free Octet platform. Recombinant anti-NP mAbs were immobilized to protein A biosensors and probed with HJs bearing $0,1,2$ or 3 copies of NP or NIP in a concentration range of $0.22-20 \mathrm{nM}$. NP-conjugated ovalbumin (Ova-NP ${ }_{15}$ ) was included as a positive control. As anticipated, Ova-NP showed strong antibody binding with little dissociation of bound protein conjugate, while no binding was detected for the naked HJ scaffold (Fig. S5A). HJ constructs bearing up to three copies of NP or NIP displayed specific and enhanced binding interactions in accordance with hapten copy number (Fig. 2E-J). Increasing the valency of hapten on the HJ from one to two resulted in an improved binding to anti-NP mAb, particularly for the low-affinity hapten NP. HJs displaying three NP or NIP molecules prompted the strongest binding profile with rapid on-rate and a slow off-rate, confirming an increased functional affinity (avidity) effect as a result of multivalent interactions. 
A

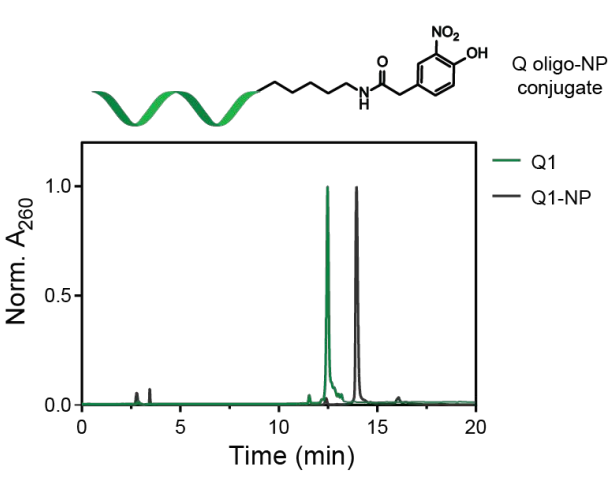

C

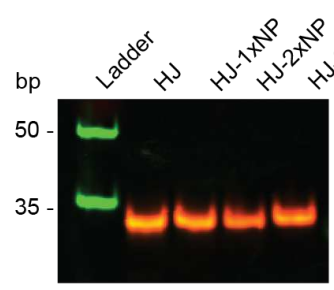

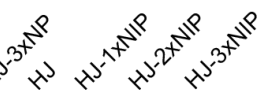
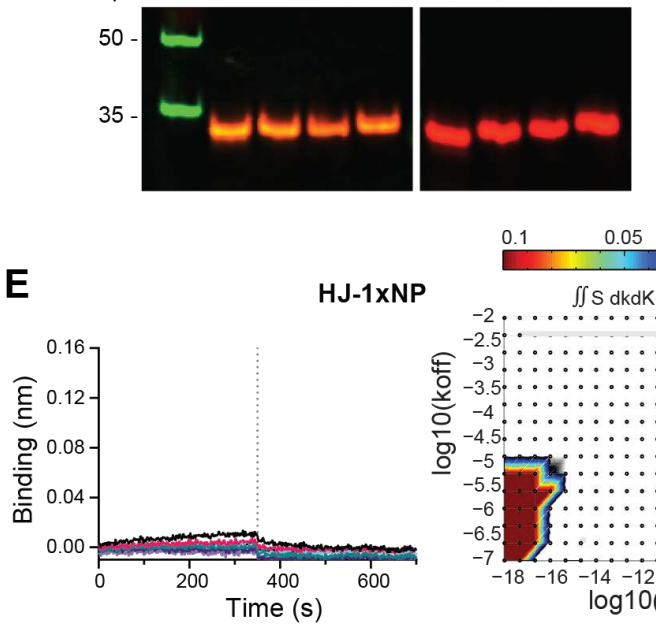

G

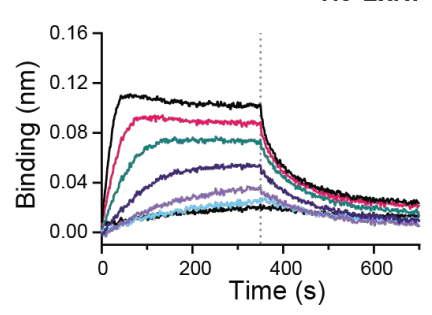

I

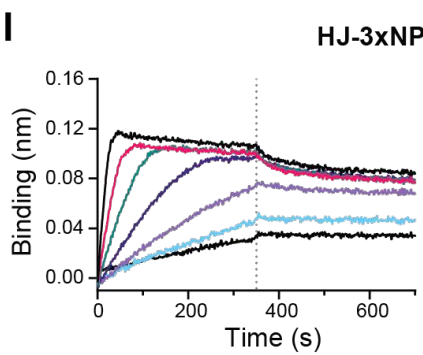

B
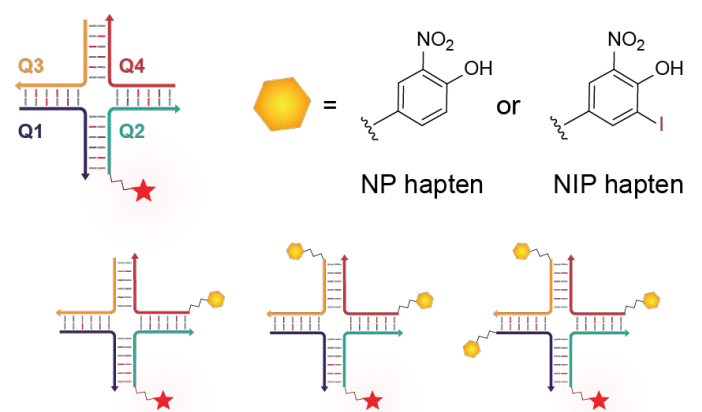

D
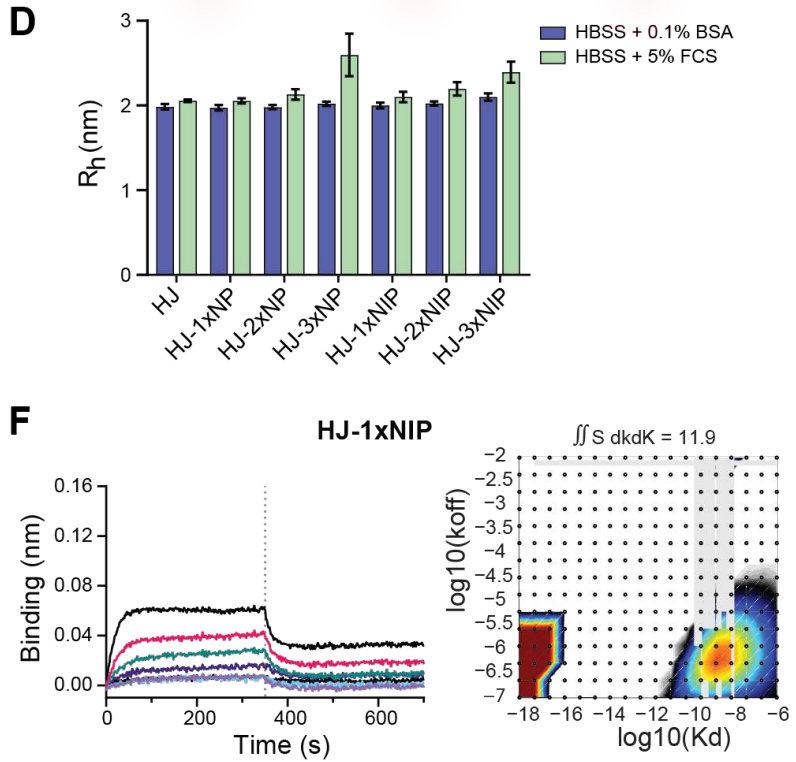

$\iint \mathrm{S} d \mathrm{kdK}=11.2 \quad \mathbf{H}$
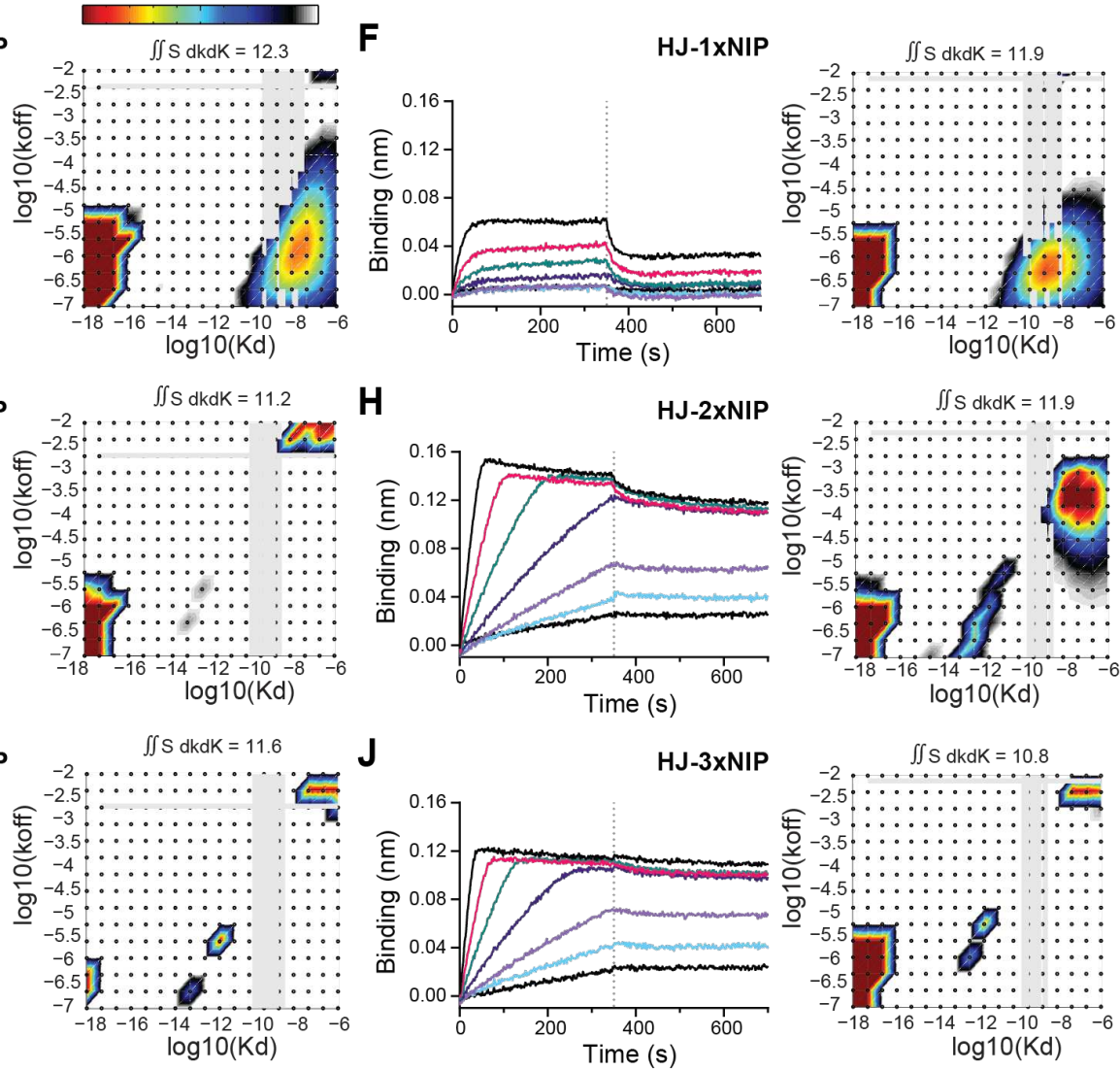

Figure 2. Assembly of hapten-functionalized HJs and their binding profile to anti-NP mAb. (A) A representative RP-HPLC chromatogram of Q1 oligo-NP conjugate purification. (B) Schematic representation of the core HJ structure and HJs bearing 1-3 NP or NIP haptens. (C) Native PAGE gel showing the assembly of HJs carrying up to three NP conjugates and a Q2 oligo bearing AF647 for detection. Green signal is SYBR Gold stain and red signal is AF647. 
Size marker used is an ultra-low range DNA ladder. (D) Apparent hydrodynamic radii $\left(\mathrm{R}_{\mathrm{h}}\right)$ of AF488-modified HJs with 0-3 units of NP or NIP (50 nM) in Hanks Balanced Salt Solution (HBSS) supplemented with $0.1 \%$ BSA (purple) or 5\% FCS (green), determined by FIDA. $\mathrm{n}=3$, error bars indicate standard deviation. (E, G, I) Bio-layer interferometry (BLI) sensorgrams indicating the binding profile of HJ-NP constructs $(0.31-20 \mathrm{nM})$ to anti-NP mAb (left panels), and modeled distributions of binding kinetics from BLI binding data for haptenated HJ constructs, derived from EVILFIT analysis (right panels). (F, H, J) Binding profile of HJ-NIP constructs (0.22-14 nM) (left panels), and modeled distributions of binding kinetics from BLI binding data for haptenated HJ constructs, derived from EVILFIT analysis (right panels). Distributions are presented as contour plots on $2 \mathrm{D}$ grids with $\log 10\left(\mathrm{~K}_{\mathrm{D}}\right)$ and $\log 10\left(\mathrm{k}_{\text {off }}\right)$ on the $\mathrm{x}$ - and $\mathrm{y}$-axes, respectively, at signal max 0.1. Binding traces and the best fits are shown in Fig. S6. Data in E-J are representative of at least 3 independent experiments with varying conditions. BSA, bovine serum albumin; FCS, fetal calf serum; NP, 4-hydroxy-3-nitrophenylacetyl; NIP, 4-hydroxy-3-iodo-5-nitrophenylacetyl.

To obtain a more quantitative evaluation of the avidity effects, we employed EVILFIT analysis $^{35,36}$, which calculates the minimal distribution in binding kinetics for heterogeneous ligand interactions (Fig. 2E-J and Fig. S6), including those produced by multivalent ligands ${ }^{37}$. EVILFIT analysis for single-NIP and single-NP HJ data were dominated by a broad distribution of interactions with $\mathrm{K}_{\mathrm{D}} \sim 10^{-9}-10^{-8} \mathrm{M}$ with matching $\mathrm{k}_{\text {off }}$ rates in the range of $10^{-7}-10^{-3.5} \mathrm{~s}^{-1}$. For bi- and trivalent NIP and NP HJs, with stronger BLI signals, we observed distributions including interactions, which seemed to better match well-defined monovalent engagements, still with $\mathrm{K}_{\mathrm{DS}}$ of $10^{-7}$ and $10^{-6} \mathrm{M}$, but with faster $\mathrm{k}_{\text {off }} \mathrm{S}$ at $\sim 10^{-2} \mathrm{~s}^{-1}$, matching what was previously reported by $\mathrm{SPR}^{34}$. Importantly, two, mostly discrete, ensembles of interactions in the range of $\mathrm{K}_{\mathrm{D}} \sim 10^{-14}-10^{-}$ ${ }^{12} \mathrm{M}$ also appeared for these multivalent ligands. Their exponential increase in affinity, differing more than 5 orders of magnitude compared with the level for the monovalent interaction, is a direct estimation of avidity based effects.

\section{Nanoscaffolded antigen displays affinity and avidity dependent binding to cognate $B$ cells}

We next investigated the binding of HJ constructs to cognate B cells using flow cytometry and confocal microscopy. To this end, we employed murine B cells purified from the B1-8hi knock-in BCR model, which carries, on one allele of its immunoglobulin heavy chain locus, the prerearranged $\mathrm{V}_{\mathrm{H}} \mathrm{D}_{\mathrm{H}} \mathrm{J}_{\mathrm{H}}$ of the $\mathrm{B} 1-8$ clone, that additionally harbors a point mutation increasing baseline affinity 40 -fold over the germline configuration ${ }^{24}$. Although most B cells in this model express the knock-in heavy chain, only a fraction of these express the VL $\lambda 1$ required for NP- 
243 binding, providing a substantial internal negative control population (Fig. S7). We estimated the 244 number of total available NP-specific BCRs a priori, based on routinely observing approximately $24512 \%$ NP-binding B cells in our untouched MACS purified B cell preparations and relying on a 246 literature derived estimate of $\sim 100,000$ BCRs per naïve, mature B cell. HJ constructs containing $2470,1,2$ or 3 haptens were incubated with B cells at a range of different stoichiometries, from 300248 fold excess of HJ:BCR down to a 1:1 ratio. Final stoichiometries were confirmed a posteori by the 249 observed NP-specific B cell frequency within each experiment. We observed B cell binding by 250 flow cytometry for all NP and NIP constructs, but not for control HJs without hapten (Fig. 3A). 251 As we approached 1:1 stoichiometric ratio between HJs and BCRs at the conditions employed, we 252 observed complete disappearance of B cell binding for monovalent NP and NIP HJ, and a shift in 253 median fluorescence intensity (MFI) for the polyvalent HJ positive B cell population. Although 254 the flow cytometric evaluation of antigen binding to BCRs on the cell surface reflects non255 equilibrium conditions, this demonstrated an affinity-, avidity- and concentration-based 256 dependence of haptenized HJ construct binding to BCRs of cognate B cells. 
A
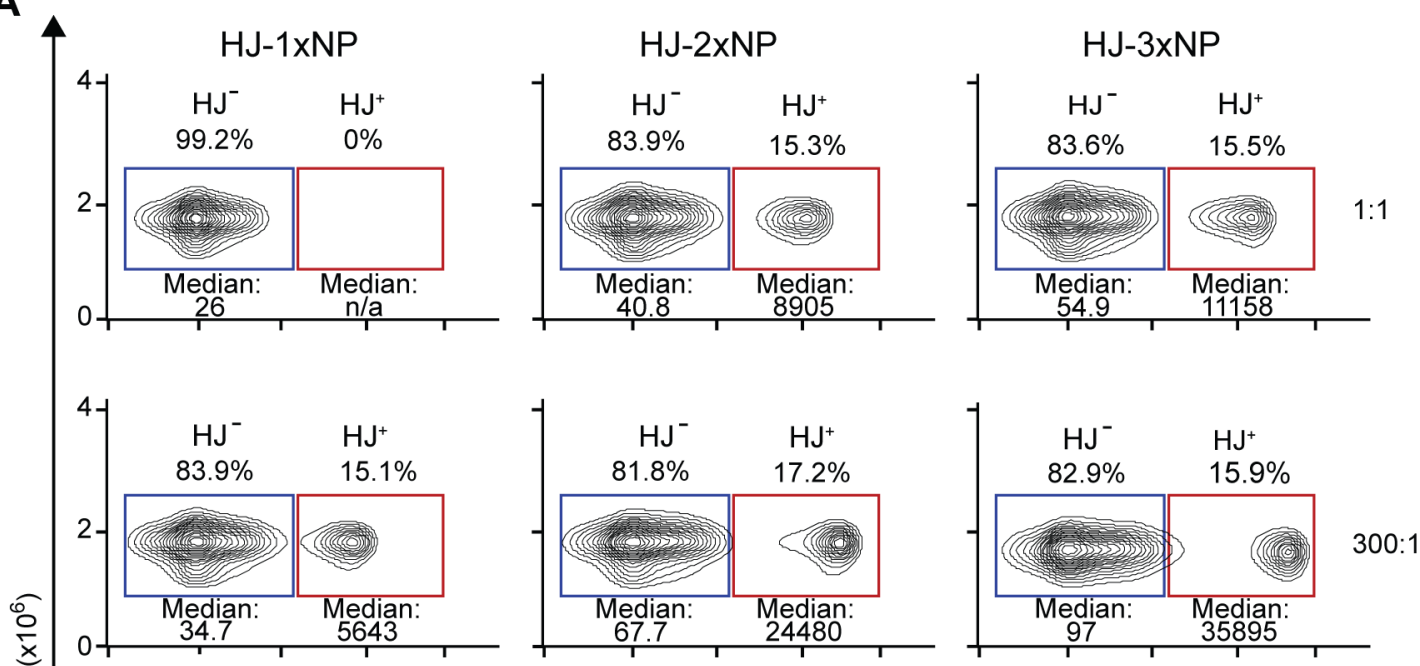

$\mathbb{1}$
0
$\mathbb{4}$
4
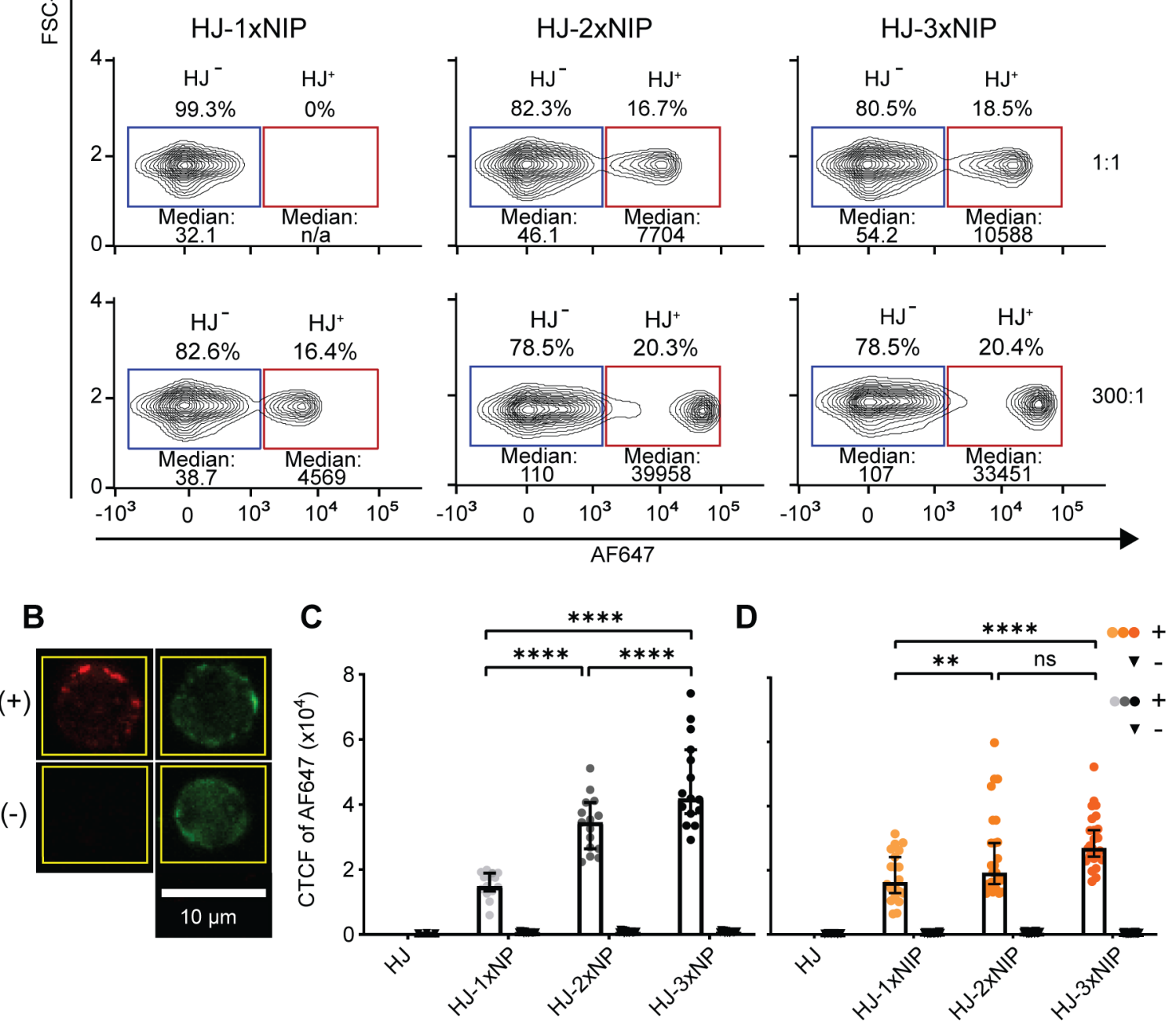
Figure 3. Binding analysis of HJ constructs with B1-8hi B cells. (A) Purified B cells from B1-8hi mice were analyzed for binding to HJ-0/1/2/3 NP or NIP using flow cytometry. Median Fluorescence Intensity (MFI) is indicated for each gate. Dilutions of HJ-0/1/2/3 NP or NIP were studied, ranging from 1:1 to 300:1 stoichiometry between HJs and BCRs. (B) Representative confocal image of cells that bound both HJ constructs and B220 (Positive, +), or B220 only (Negative, -). The binding of HJs is indicated by AF647 signal (red), whereas the binding of B220 is indicated by green signal (FITC). The images show the same field of view, with one positive and one negative cell. (C, D) B cells that bound HJ constructs were analyzed based on Corrected Total Cell Fluorescence (CTCF) of AF647 fluorescence signal within the area in the yellow square $(n=15)$. Each dot indicates an individual cell, and bars indicate median values. Statistical analysis was done using two-way ANOVA with Tukey's multiple comparisons test, ${ }^{* *} \mathrm{p}=0.0049,{ }^{* * * *} \mathrm{p}<0.0001$.

To further validate the binding, we imaged the B cells using confocal microscopy, after exposure to the HJ constructs (Fig. 3B). The AF647 signal from HJ-bound cells was measured and processed using corrected total cell fluorescence (CTCF), then internally compared to B cells that did not bind HJs. Naked HJs did not bind B1-8hi B cells, whereas HJs carrying a single hapten yielded a weaker signal compared to polyvalent HJs. The results indicated a statistically significant increase in AF647 signal, which correlated with the number of haptens per HJ, with 3 haptens yielding the highest signal (Fig. 3C and D).

Taken together, our flow cytometric and confocal imaging analyses demonstrated discrete, titratable binding of hapten-conjugated HJ constructs to the B1-8 subpopulation, correlating with affinity and valency.

\section{Antigen exerts agonistic effects on the BCR as a function of increasing affinity and avidity}

Having observed concentration-dependent and specific binding of monovalent as well as polyvalent hapten-HJ conjugates to B1-8hi B cells, we next asked to what extent this binding induced B cell activation. To this end, we used ratiometric calcium flux analyses based on the calcium-sensitive fluorochromes Fluo-3 and Fura Red. Again, we estimated the relative stoichiometries of antigen to BCR and titrated HJs across a range of stoichiometries from supraphysiological excess (200:1) to below equimolar stoichiometry (0.2:1). Importantly, calcium flux analyses were carried out under near-equilibrium conditions in the absence of washing steps, and moreover, based on the AF647 signal, we could follow the binding of HJs simultaneously with reading out the calcium flux (Fig. 4A). At high stoichiometries of HJs, both polyvalent and 
292 monovalent constructs caused calcium influx, resulting in a change of Fluo-3 to Fura Red signal 293 ratio and indicating cellular activation (Fig. 4B). Furthermore, both types of haptens activated the 294 cells, with NP activation being weaker than NIP as expected from their known affinity difference. 295 When the ratio of HJs to BCRs was lowered to approximately 20:1, the addition of monovalent 296 HJs did not result in B cell activation (Fig. 4C). The signal for HJ-1xNIP was below background 297 and no signal was detectable for HJ-1xNP. Importantly, this was seen under conditions where 298 equilibrium calculations still indicated a significant degree of receptor occupancy by HJ-1xNIP, 299 on the order of approximately 14,000 BCRs vs. 94,000 BCRs, based on 2,000:1 vs. 20:1 300 stoichiometry of HJ:BCR. Of note, this was based on the specific conditions of our assay, i.e., 31 301 vs. $0.31 \mathrm{nM} \mathrm{HJ}$ in-well to an estimated 16/32 pM BCR/Fab available based on 200,000 B cells 302 with 100,000 BCRs per cell, 2 Fab per BCR, 10\% NP-specific B cells, and using an affinity 303 constant of B1-8hi for NIP of $\mathrm{K}_{\mathrm{D}}=1.9 \times 10^{-9} \mathrm{M}^{34}$. The determinant of binding is the relative 304 concentration of antigen to Fab and the $\mathrm{K}_{\mathrm{D}}$ of the specific interaction, but for simplicity, we refer 305 to stoichiometry under standard conditions with a fixed number of available Fab arms for each 306 model studied.

307 Together with our binding studies, our calcium flux analyses suggested that at more 308 physiologically relevant levels, monovalent antigens can be bound by B cells through their BCRs, 309 yet fail to activate the cells. At supraphysiological concentrations of monovalent antigen, however, 310 B cell activation did occur. 

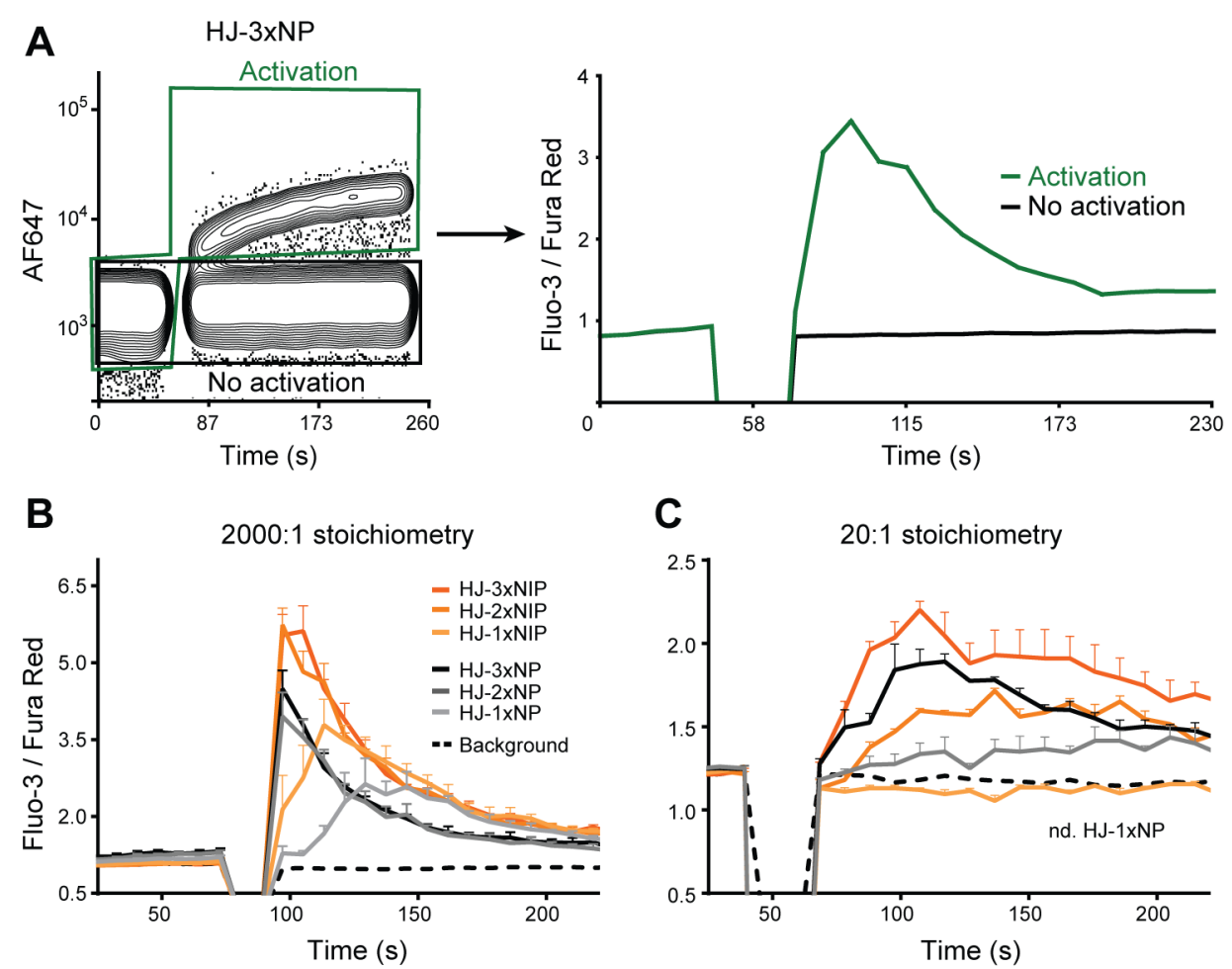

Figure 4. Activation of B1-8hi B cells with HJ constructs. (A) B1-8hi B cell activation was studied in terms of calcium flux by flow cytometry. Left, binding of HJ followed by AF647 signal. Right, calcium flux of cells upon

314 stimulation with HJ-3xNP in the HJ binding (activation) vs. non-binding (no activation, i.e., background) population.

315 (B) HJ constructs with various valencies (0, 1, 2 or 3$)$ of two types of haptens (NP or NIP) at high stoichiometry of 316 HJ constructs to BCR (2,000:1). (C) as B, but at low stoichiometry (20:1). In B and C, mean \pm SD of 3 independent 317 experiments are shown.

\section{No qualitative difference between activation induced by mono- and polyvalent antigen}

In light of earlier findings that monovalent antigen engagement of the B cell receptor could induce receptor activation, but failed to promote subsequent antigen presentation ${ }^{14}$, we sought to qualify the observed activation of B cells that occurred with poly- as well as monovalent HJ at supraphysiological antigen levels. To this end, we interrogated the actin-mediated polarization, capping and receptor-mediated endocytosis that occurs downstream of BCR activation using imaging flow cytometry (Fig. 5 and Fig. S8). To generate a baseline for surface bound HJ, we incubated B1-8hi cells with HJ constructs on ice, allowing binding but precluding energydependent internalization (Fig. 5A, bottom). To evaluate the antigen-driven uptake and internalization, cells were incubated at $37^{\circ} \mathrm{C}$ for 10 or 60 min after addition of $\mathrm{HJ}$ constructs. At 
10 min, a clear "capping" phenomenon was observed, where HJs localized in clusters on the cell 330 surface. After 60 min, HJs were increasingly internalized by B cells (Fig. 5). We quantified the 331 ratio between AF647 signal within "membrane" and "internalized" masks to compare how well 332 different $\mathrm{HJ}$ constructs were taken up by B cells at low or high stoichiometries. Polyvalent constructs, particularly HJ-3xNIP, were internalized to the greatest degree at both 1:1 and 300:1 stoichiometries. However, even the monovalent NIP-HJ was internalized to an appreciable extent following incubation at supraphysiological stoichiometry. This indicated that there was no fundamental qualitative difference between BCR activation induced by poly- and monovalent $\mathrm{HJ}$ 337 antigen.

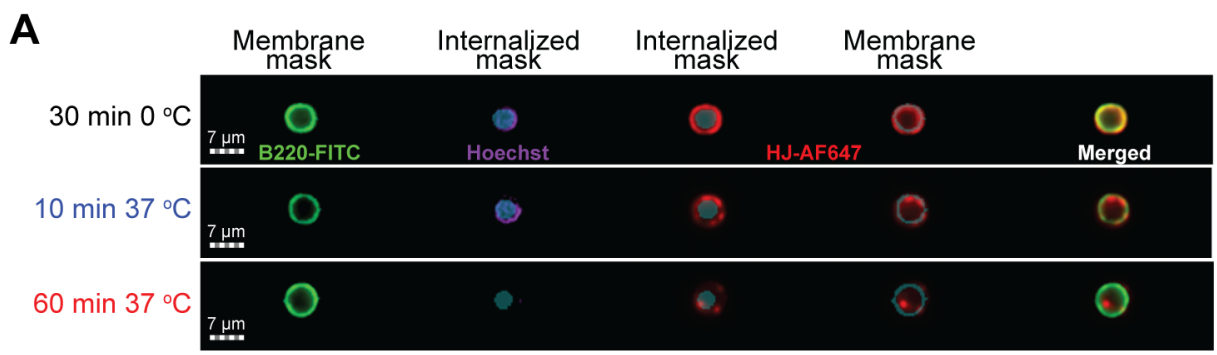

B
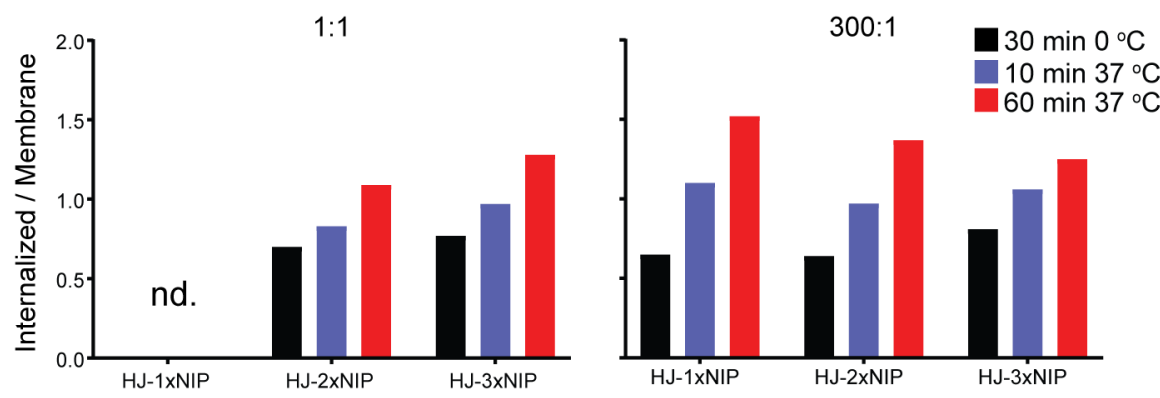

Figure 5. Uptake and internalization of HJs by B1-8hi B cells measured by imaging flow cytometry. (A) Representative images from imaging flow cytometry analyses of uptake and internalization of HJs by B1-8hi B cells. A masking method was used to measure the amount of HJ signal (red) on the surface of the cells and within the cells. Membrane and internalization masks were made based on the B220 marker (green), either overlapping with it or excluding it. Nuclear Hoechst stain (blue) shows the potential overlap of internalized mask with the nucleus. (B) AF647 signal from different HJ constructs within these masks was quantified across 3,000-5,000 individual cells and the median ratio of internalized/membrane signal was plotted for different hapten valencies, HJ:BCR stoichiometries, as well as incubation conditions (time and temperature). Raw distributions for membrane/internalized signals are shown in Fig. S7. 


\section{Supraphysiological amounts of micromolecular antigens fail to activate B cells}

351 One explanation for the BCR activation occurring at high concentrations of monovalent haptenHJ constructs could be the appreciable size and potentially the surface charge of the HJs, which could change the microenvironment surrounding engaged BCRs. To investigate this possibility, we examined the ability of micromolecular antigens to induce activation. To be able to assess activation independently of our ability to monitor antigen-binding, we turned to the B1-8i kappa light chain knock-out mouse model (B1-8i JK knock-out) ${ }^{38}$. In this model, approximately 50\% of the B cells carry receptors specific for NP and NIP, albeit with somewhat lower affinity than B18hi cells (approximately 40-fold decreased).

Because B1-8i kappa knock-out mice carry homozygous knock-in of the B1-8i heavy chain, as opposed to the single knock-in of the B1-8hi model and the single recombined heavy chain in wild-type B cells enforced through allelic exclusion, it was possible that they displayed twice the number of BCRs normally present on the B cell surface. Therefore, we examined their surface expression of key cell surface molecules independent of (B220/CD45R), linked with (CD19, signaling component of CD21 co-receptor) or representative of the BCR (CD79 and IgD), compared to either B1-8hi B cells or wild-type C57B16/J B cells. B1-8hi cells had slightly lower MFI for IgD than $\mathrm{C} 57 \mathrm{Bl} / 6$ and $\mathrm{B} 1-8 \mathrm{i}$ cells, which were identical, and no other significant differences were observed (Fig. S9). Although flow cytometric evaluation of membrane receptor levels is not absolutely quantitative, the near-identical signals observed indicated a similar level of expression of the BCR irrespective of the knock-in or wild-type provenance of the receptor.

Having established that the level of BCRs displayed by B1-8i cells is in line with that of wild-type B cells, we set up an experiment to interrogate the activation potential of small-molecule hapten antigens. NIP-OH and NIP-caproic acid (Fig. 6A) were titrated onto B1-8i cells and we measured the calcium flux potential. This revealed that even at 200,000-fold molar excess, these antigens were unable to induce activation (Fig. 6B and C). Even considering the lower affinity of B1-8i B cells, these conditions should cause $>99 \%$ BCR occupancy, corresponding to $\sim 99,400$ engaged BCRs on the cell surface of cognate B cells at the conditions employed. This was based on $16 \mu \mathrm{M}$ NIP hapten, 80/160 pM BCR/Fab available based on 200,000 B cells with an estimated 100,000 BCRs per cell, 2 Fab per BCR, an observed 50\% NP-specific B cells, and using an affinity constant of B1-8i for NIP of $K_{D}=1 \times 10^{-7} \mathrm{M}$. Importantly, these results, obtained under conditions where a 
high fraction of BCRs would be occupied, indicated that antigen binding, in and of itself, was insufficient to drive BCR activation.

We next considered the possibility that the observed difference between monovalent hapten HJ conjugates and isolated small-molecular weight haptens could be explained by a difference in propensity to aggregation. Despite the documented monodispersity of HJ scaffolds, and our data demonstrating that they do not aggregate even in complex environments (serum), it is virtually impossible to exclude that minute levels of aggregates could be formed in situ. To evaluate the likeliness of aggregate formation explaining the observed activation effects, we titrated HJ-2xNIP and determined the minimal stoichiometry that could induce activation and arrived at a ratio around 2:1 HJ:BCR (Fig. 6D). We then determined the minimal stoichiometry for 1xNIP-HJ that could induce activation and arrived at around 20:1. Hence, the 1x conjugate was 10-fold less potent in inducing activation (Fig. 6E). If the activity of the $1 \mathrm{x}$ conjugate should be explained by aggregate formation, this would require aggregates equivalent of $10 \% 2 \mathrm{x}$ conjugate, something which could be ruled out by our FIDA analyses (Fig. 2 and Fig. S3). Taken together, this demonstrated that aggregate formation in situ is not the driving force behind the observed activation by monovalent antigen.

To further delineate the minimal requirement for monovalent antigen to exert an agonistic effect on the BCR, we generated a double-stranded variant of the Q4 oligo component of the HJ, by complexing the Q4 oligo conjugated with a single NIP to its complementary DNA strand. Importantly, this construct would have half the mass of the HJ nanoscaffold, $\sim 8 \mathrm{kDa}$, and carry a single NIP. Yet, due to the extended linear conformation of the double-helix, as opposed to the cruciform shape of the HJ, combined with the rotational freedom around the hapten linkage, we expect this nanoscaffold to occupy a minimal footprint around the Fab binding site, insufficient to drive activation. Congruent with this notion, we did not observe any activation with the Q4 oligo at ratios severalfold exceeding the minimal activating level of HJ-1xNIP (Fig. 6F). 
A

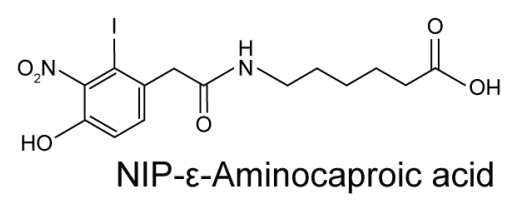<smiles>O=C(O)Cc1ccc(O)c([N+](=O)[O-])c1I</smiles>

B $\mathrm{NIP}-\mathrm{OH}$

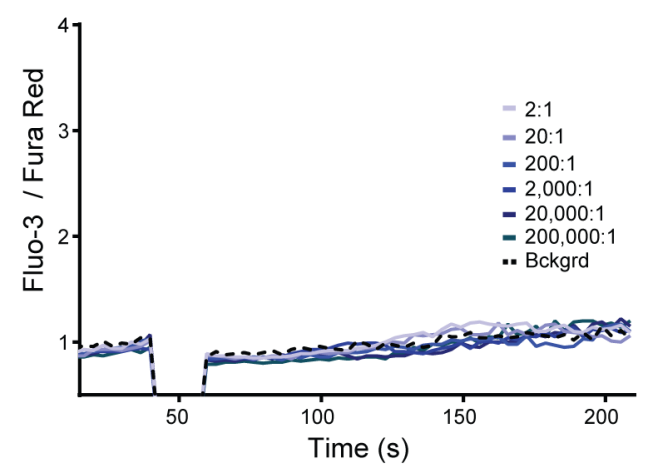

D HJ-2xNIP titration
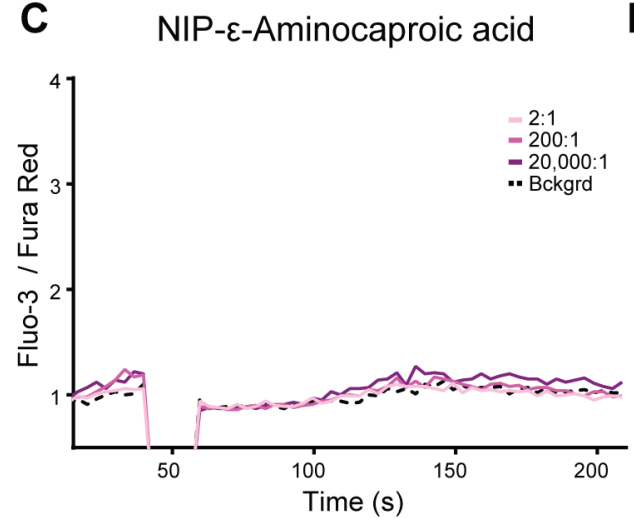

E HJ-1xNIP titration

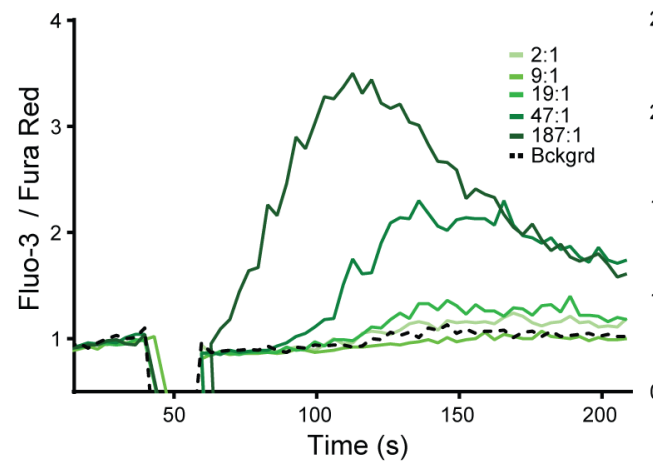

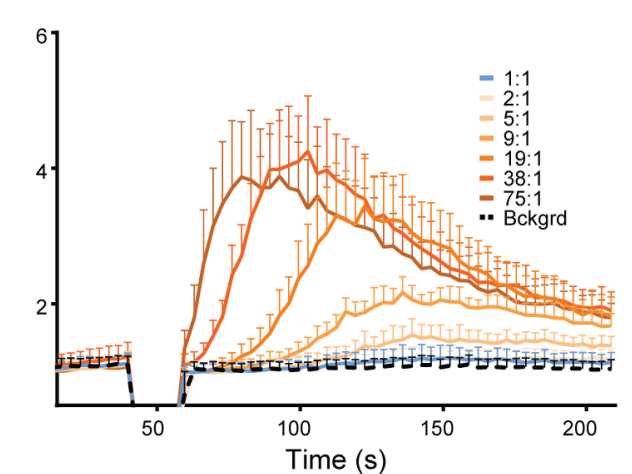

F

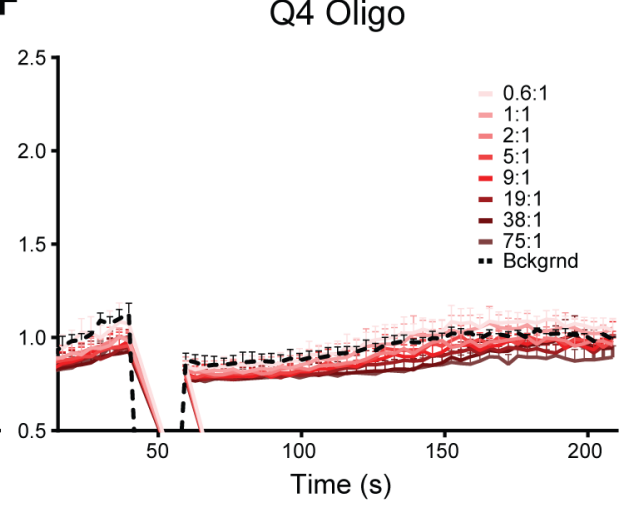

Figure 6. Activation of B1-8i B cells with small-molecule haptens versus HJ constructs. (A) Chemical structure of NIP-E-Aminocaproic acid and NIP-OH. (B) Calcium flux of cells upon stimulation with NIP-OH at varying molar stoichiometries from 200,000-fold molar excess of antigen to BCR, down to 2:1 stoichiometry. (C) Calcium flux of cells upon stimulation with NIP- $\varepsilon$-aminocaproic acid at varying molar stoichiometries from 20,000-fold molar excess down to 2:1 stoichiometry. (D) Calcium flux of cells upon stimulation with HJ-2xNIP at varying molar stoichiometries from 75-fold molar excess of HJ to BCR down to 1:1 stoichiometry. (E) Calcium flux of cells upon stimulation with HJ-1xNIP at varying molar stoichiometries from 187-fold molar excess of HJ to BCR down to 2:1 stoichiometry. (F)

413 Calcium flux of cells upon stimulation with Q4 oligo conjugated to a single NIP and complexed with its 414 complementary strand, at varying molar stoichiometries from 75-fold molar excess of Q4 oligo to BCR down to 0.6:1 
415 stoichiometry. In all panels, background refers to naked HJ stimulation. In C and E, mean \pm SD of 3 independent 416 experiments are shown, whereas in A, B and D, representative data from one of two experiments are shown. 


\section{Discussion}

418 The B cell receptor is, arguably, the most versatile cellular receptor: it is generated by somatic recombination to potentially recognize any ligand of virtually any origin, even ligands that never existed in nature, and it is malleable through somatic hypermutation. This versatility and diversity are likely the main reasons why it has remained a significant challenge to firmly define the molecular mechanism of BCR activation. Our findings here address two fundamental questions, as set out in the introduction: 1) What is the resting state distribution of BCRs on the B cell surface, and 2) What are the minimal requirements for antigen-driven activation of the BCR?

To address the first question, we employed DNA-PAINT. Of note, previous attempts to determine BCR distributions on the cell surface have been made, however, classical super-resolution approaches are prone to quantification artefacts such as under- or overcounting due to the unpredictable photophysical blinking properties of fluorescent proteins or organic dyes ${ }^{39,40}$. Prior studies employing PALM and (d)STORM to provide plasma membrane maps of the BCR and associated components more or less invariably reported nanoscale clusters or larger protein islands ${ }^{13,41,42,43}$. However, similar observations were made for virtually any membrane protein ${ }^{44}$, and it has emerged that multiple observations of single fluorophores also lead to clustered localizations that may closely resemble clustered molecules. In turn, this impedes direct inference of the presence of protein clusters based on the observed localization clusters. We circumvented this obstacle by employing DNA-PAINT, which is able to overcome these limitations. Our data indicate that BCRs are not significantly pre-clustered in an organized fashion, as has otherwise been supposed in the dissociation-activation model ${ }^{45}$. Our findings regarding the BCR distribution are, in essence, a corollary to the observation that apparent $\mathrm{T}$ cell receptor (TCR) nanoclustering could be attributed to overcounting artifacts inherent to single-molecule-localization microscopy and that both resting naïve and antigen-experienced $\mathrm{T}$ cells display monomeric TCR distributions ${ }^{46,47}$. Based on our DNA-PAINT analyses, we estimated that there was an average of 10,000 BCRs per naïve B cell, which is significantly below a literature derived estimate of 100,000 receptors per cell. Because the labeling efficiency is likely below $100 \%$, we do consider our value to be somewhat underestimated. However, the discrepancy may also be explained in part by species differences between human and murine B cells. Importantly, in our subsequent equilibrium 
calculations, we conservatively relied on the literature derived BCR estimate, precluding the possibility of overestimating ligand:receptor ratios.

To address the second question, we employed a nanoscaffold allowing precise stoichiometric assemblies of defined antigenic valency, in a setting that allows precise determination of antigen:BCR ratios. We found that both monovalent and polyvalent configurations could induce activation, although monovalent antigen only exerted an activity at what are likely supraphysiological conditions. When activation occurred, there was no qualitative difference between the activation elicited by mono- and polyvalent antigens. For comparison with our nanoscaffolded antigens, we probed the ability of simple, small-molecule haptens to induce activation, and found that they failed to do so, even at supersaturating conditions. Two central points can be drawn from our observations: firstly, it is not the binding itself, which induces activation; and secondly, at certain conditions monovalent antigens are able to induce activation, albeit inefficiently. Our results reconcile prior conflicting data in the literature, where monovalent antigen has variably been assigned either activating ${ }^{14,15,48}$ or non-activating ${ }^{19,20}$ capacity, as condition-dependent observations that could, in isolation, support either claim. At the same time, our findings upend this discussion of antigenic activity by the observation that not only affinity and valency, but also additional molecular properties such as size are critical in determining their capacity for BCR activation.

The activity of larger monovalent antigens is highly concentration-dependent, and robust activation requires what is arguably supraphysiological levels, although on a side note, it is not really known what the antigen availability is in vivo, and what range of relative ratios of antigen to cognate BCRs is encountered in the physiological setting. In our in vitro setting, monovalent antigen activity required molar stoichiometries that indicated engagement of a significant number of BCRs at the conditions employed. Notably, in the experiments employing B1-8hi cells, we did not see activation with HJ-1xNIP at 20:1 stoichiometry, whereas we did in the case of B1-8i cells. The difference of these two scenarios is $\sim 10 \%$ vs. $\sim 50 \%$ binders, and $\mathrm{K}_{\mathrm{D}}$ 's of $1.9 \times 10^{-9} \mathrm{M}$ and $1 \times 10^{-7} \mathrm{M}$ for B1-8hi and B1-8i, respectively. Equilibrium calculations reveal engagement of 14,610 and 1,630 BCRs, respectively, for these two scenarios. In light of this, the lack of observable activation in the case of B1-8hi cells is somewhat surprising, and likely an artefact caused by our inability to accurately identify the small $(\sim 10 \%)$ subpopulation that is fluxing 
calcium, within the sizeable non-fluxing background. The B1-8i results, however, demonstrate that monovalent antigen can induce activation, and notably can do so under conditions where only a fraction (1-2\%) of BCRs are engaged. This result is unlikely to be a consequence of in situ aggregation, because the activity of our monovalent antigen preparation was comparable to a 10 times more diluted divalent antigen preparation. Hence, if the activity of the monovalent antigen preparation should be solely explained by a contaminant fraction of aggregate, this should make up at least $10 \%$ of the material, and this was easily ruled out by our FIDA analysis. In further support of this, we did not observe any multivalency effects in our BLI and EvilFit analyses of the monovalent antigen preparations.

The 20:1 scenario for NIP-HJ and B1-8i cells also hints that it is unlikely that the activity depends significantly on individual receptor occupancy time, because the relatively low affinity and the low number of BCRs engaged at equilibrium would indicate a continuous unbinding and binding of the monovalent antigen. This notion, combined with the size requirement and demonstration that binding in and of itself is insufficient, suggests that occupation of the BCR with a sizeable antigenic binder is intimately linked with activation.

These findings portray a steric aspect of binding large antigens as central in driving BCR signaling, which also gives new insight on the role of polyvalency for at least some antigens in BCR signaling. The binding kinetic studies clearly revealed that our polyvalent constructs generated an interaction pattern characteristic for other polyvalent molecules, with multiple, discrete ensembles of interactions in spite of their structural homogeneity ${ }^{49}$. This confirms that polyvalent interactions are permitted by the constructs, but comparison with the monovalent antigen excludes that structural properties of the polyvalent interaction, namely clustering, per se, is critical for the increased signaling. In their thermodynamic model of polyvalent interaction, Kitov and Bundle used a step-wise description with one initial intermolecular interaction, followed by multiple intramolecular interactions ${ }^{50}$. The latter would form more easily, once the polyvalent construct has made its initial transition from a soluble to a surface tethered form. In this way, the polyvalency of the constructs can drive additional BCR interactions to a level, which can be mimicked by a monovalent construct at a much higher concentration. Yet in both cases, the critical event for signaling is the engagement between a single antigen and BCR. Our finding that soluble 
monovalent antigen can activate the BCR argues strongly against the simple cross-linking model found in most textbooks, as this allows for only multivalent activities.

The notion that bulky soluble, or membrane-bound monovalent antigen affords activation, whereas micromolecular antigen binding does not, bears a striking resemblance to the scenario of $\mathrm{T}$ cell activation, which invariably occurs on membrane surfaces. Although the classical model for T cell activation is also based on clustering ${ }^{51}$, an alternative hypothesis is embodied in the kinetic segregation model ${ }^{52}$. The kinetic segregation model posits that under resting conditions, the kinase activity on CD79 ITAMs is counterbalanced by the highly prevalent phosphatase CD45. However, TCR binding to cognate peptide:MHC on an opposing cell surface entails a close approximation of membranes due to the short-range TCR - peptide:MHC interactions. This excludes the extended and bulky CD45 molecule from the nascent synapse and shifts the local balance in favor of kinase activity, thereby initiating the signaling cascade. This mechanism could easily be extrapolated to BCR sensing of membrane-scaffolded antigen, e.g., on follicular dendritic cells. However, it would only be applicable to monovalent and multivalent soluble antigens on the condition that these were bulky enough, or had other properties that allowed them to occlude CD45 from the vicinity of the BCR. In an evolutionary perspective, a common cell biological principle could well govern the mechanism of activation of the TCR and the BCR, despite the inherent differences in the breadth and nature of antigens recognized. However, in its current formulation as a model of $\mathrm{T}$ cell activation, the kinetic segregation model requires a juxtaposing cell membrane to exclude CD45 from the signaling complex, and our data are more congruent with something occurring in a much smaller environment.

Thus, taken together, our data point to a unique mechanism of antigen-driven activation through the BCR. Based on the speed with which activation occurs, and the overall congruence of our observations with the kinetic segregation model of $\mathrm{T}$ cell activation, we suggest that the B cells are poised for activation in a kinetic equilibrium through the counterbalanced activities of kinases and phosphatases, allowing a relatively small perturbation to rapidly shift the balance in favor of activation. The critical event for signaling is the engagement between a single antigen and BCR. However, the lack of activation by micromolecular antigens, under conditions where a high fraction of BCRs are occupied, demonstrates that antigen binding, in and of itself, is insufficient to drive activation. Our finding that a monovalent $\sim 16 \mathrm{kDa}$ antigen can induce activation, while a 
535 monovalent $\sim 8 \mathrm{kDa}$ antigen cannot, demonstrates that the antigen needs to occupy a certain 536 footprint around the Fab binding site to trigger activation. This is congruent with our DNA-PAINT 537 imaging results, supporting that the BCR microenvironment rather than physical BCR interactions 538 are responsible for regulating its nanoscale organization.

539 Our revised model for BCR activation accommodates both monovalent and multivalent antigen 540 activities. Furthermore, it explains qualitative differences between the two in terms of the 541 thermodynamic model of polyvalent interaction rather than simple clustering. This enables a more 542 profound understanding of the complex activities of soluble antigens in their native form and 543 subsequent to antigen scaffolding on membranes. Soluble antigen recognition is arguably the 544 simplest scenario of $\mathrm{B}$ cell activation, and that which sets it apart from $\mathrm{T}$ cell recognition of peptide $545 \mathrm{MHC}$; yet in vivo, the BCR activation mechanism must at the same time accommodate antigen 546 scaffolding on membranes, such as the surface of follicular dendritic cells ${ }^{20,53,54}$. The notion that 547 size and valency are important determinants of antigenic activity is additionally well in line with 548 the evolutionary drive to recognize larger particulate material, such as virus particles or bacteria, 549 and to be ignorant of smaller, likely innocuous antigens. It is also congruent with the long-standing 550 notion that haptens do not elicit immune responses in and of themselves. The higher potential of 551 repeated ligands can be rationalized in terms of the common properties of biologically relevant 552 antigens: sizeable particles with repeated structures, such as the repeated carbohydrate structures 553 of the glycocalyx or spike proteins of the virion. Yet the capability to respond to sizeable 554 monovalent antigen averts vulnerabilities in the B cell defense. 


\section{Materials and methods}

556 Sequences

557

Q1: 5'-NH ${ }_{2}-\mathrm{C} 6-\mathrm{mC}+\mathrm{CmG}+\mathrm{TmCmCmT}+\mathrm{GmA}+\mathrm{GmCmC}-3^{\prime}$

558

Q2: 5'-NH $2-\mathrm{C} 6-\mathrm{mCmA}+\mathrm{CmA}+\mathrm{GmTmG}+\mathrm{GmA}+\mathrm{CmGmG}-3^{\prime}$

559

Q3: 5'-NH ${ }_{2}-\mathrm{C} 6-\mathrm{mG}+\mathrm{GmC}+\mathrm{TmCmAmCmC}+\mathrm{GmA}+\mathrm{TmC}-3^{\prime}$

560

Q4: 5'-NH $2-\mathrm{C6}-\mathrm{mGmA}+\mathrm{TmC}+\mathrm{GmGmAmC}+\mathrm{TmG}+\mathrm{TmG}-3^{\prime}$

561

562

LNA and 2'-OMe RNA nucleotides are indicated with “+”" and “m”, respectively. Q1, Q2, Q3, and

563

Q4 oligos were synthesized with a 5' amino modification via a carbon 6 (C6) linker by the lab of

564 Jesper Wengel, University of Southern Denmark, as previously described ${ }^{23}$.

565

\section{Conjugation reactions}

567

Q oligos were conjugated to the following two haptens 4-hydroxy-3-nitrophenylacetyl-O-

568 succinimide ester (NP-OSu) and 4-hydroxy-3-iodo-5-nitrophenylacetyl amino caproyl acetyl-Osuccinimide ester (NIP-eCAP-OSu) (Santa Cruz Biotechnology) or Alexa Fluor 647 NHS ester (Thermo Scientific). The reactions were carried out in $100 \mathrm{mM}$ HEPES (pH 8.2), 40\% DMSO in a 40-fold excess of the NHS ester at $25^{\circ} \mathrm{C}, 500 \mathrm{RPM}$ shaking for $4 \mathrm{~h}$.

572

573

\section{Reverse-Phase HPLC purification}

574 All reaction products were ethanol precipitated, reconstituted in nuclease free water and 575 subsequently purified by RP-HPLC (Agilent) on a Phenomenex Evo C18 reverse phase column 576 using a $\mathrm{MeCN}$ gradient starting from 5\% MeCN, 5\% triethylammonium acetate (TEAA) to 95\% MeCN over 25 min followed by 6 min with 95\% MeCN. Based on UV detection at wavelengths

$578260 \mathrm{~nm}$ and $430 \mathrm{~nm}$ the fractions holding the reaction products were collected and lyophilized, 579 and reconstituted in nuclease free water.

\section{Electrophoretic mobility shift assays}

582 Reaction products were analyzed using polyacrylamide gel electrophoresis (PAGE). Denaturing 583 PAGE gels (12\%) were prepared by mixing $17 \mathrm{~mL}$ UreaGel Diluent (National Diagnostics), 17 $584 \mathrm{~mL}$ UreaGel Concentrate (National Diagnostics), and $4 \mathrm{~mL}$ 10x Tris-Borate EDTA (TBE) (Gibco 585 by Life Technologies), $320 \mu \mathrm{L}$ ammonium persulfate (APS) (1\%), and $16 \mu \mathrm{L}$ 
tetramethylethylenediamine (TEMED), both purchased from Sigma Aldrich. Gels were prerun for $30 \mathrm{~min}$ at $20 \mathrm{~W}$ before loading the samples. Native PAGE gels (12\%) contained $12 \mathrm{~mL}$ AccuGel 29:1 (40\%) (National Diagnostics), $2 \mathrm{~mL}$ 10xTBE, $26 \mathrm{~mL}$ MilliQ, $320 \mu \mathrm{L}$ APS, and $16 \mu \mathrm{L}$ TEMED. All PAGE gels were stained with $5 \mu \mathrm{L}$ SYBR Gold (Invitrogen) in $200 \mathrm{~mL}$ MilliQ water for $15 \mathrm{~min}$ and scanned on an Amersham Typhoon 5 scanner (GE Healthcare). An Ultralow range DNA ladder (10-300 bp, Thermo Scientific) was used as a size marker. NuPAGE 4-12\% Bis-Tris SDS-PAGE gel (ThermoFisher) was run in 1xMES buffer (ThermoFisher) at $150 \mathrm{~V}$ for $40 \mathrm{~min}$. Subsequently, the gel was stained in Coomassie Brilliant Blue for $1 \mathrm{~h}$ and destained in MilliQ water $\mathrm{O} / \mathrm{N}$ before scanning on a GelDoc Ez Imager (Bio-Rad).

\section{Liquid chromatography mass spectrophotometry (LC-MS)}

LC-MS analysis was conducted to find the mass and assess the purity of the conjugates. This was done by analyzing 40 pmol of each conjugate using a Shimadzu LCMS-2020 (ES) coupled to a Shimadzu Prominence RP-UPLC system equipped with a Phenomenex Gemini $3 \mu \mathrm{m}$ C18 column $100 \times 4.6 \mathrm{~mm}$ in a gradient of MeOH in HFIP (100 mM)/TEA (80 mM) buffer. HFIP, 1,1,1,3,3,3hexafluoro-isopropanol; TEA, trimethylamine.

\section{Assembly of $\mathrm{HJ}$ constructs}

The assembly of HJs was conducted by mixing equimolar amounts of each oligo in PBS or in binding buffer for the kinetic studies. In a Thermal Cycler (Eppendorf), the samples were heated up to $70^{\circ} \mathrm{C}$ and cooled down to $4^{\circ} \mathrm{C}$ by following a linear temperature ramp over $90 \mathrm{~min}$.

\section{Bio-layer interferometry binding assay}

Binding studies of NP/NIP-functionalized HJs were carried out using an Octet platform (FortéBio). Protein A biosensors (FortéBio) were used for the immobilization of recombinant mouse monoclonal anti-NP antibodies (Abcam; ab206523) diluted in binding buffer (1x PBS, 0.02\% Tween-20, 0.1\% BSA, pH 7.4). The biosensors were pre-hydrated in binding buffer for a minimum of 10 min prior to coating with the anti-NP mAbs $(1 \mu \mathrm{g} / \mathrm{mL})$ through the interactions of protein A with the Fc region of the mAb. To remove excess anti-NP mAb, the sensors were washed once in binding buffer. The binding of anti-NP mAb to HJ constructs bearing 0-3 NP or NIP molecules was conducted using a 2-fold serial dilution ranging from $20 \mathrm{nM}$ to $0.2 \mathrm{nM}$. The association and 
617 dissociation steps lasted 350 and $500 \mathrm{~s}$, respectively. Regeneration of sensors occurred for $2 \times 5 \mathrm{~s}$

618 in $10 \mathrm{mM}$ glycine (pH 1.7).

619

620

\section{D binding kinetic analysis}

621 The two-dimensional fits were made on the MATLAB 2012a platform (Mathworks) using the 622 fitting tool EVILFIT version 3 software created by Peter Schuck as previously described ${ }^{55,56}$. In 623 brief, input values matched the start and end injection time and included concentrations spanning 624 from $219 \mathrm{pM}$ to $20 \mathrm{nM}$. The association phase was fitted from $\mathrm{t}=$ "injection start" plus $1 \mathrm{~s}$ to $\mathrm{t}=$ 625 "injection end" minus $5 \mathrm{~s}$. Dissociation phase was fitted from $\mathrm{t}=$ "injection end" plus $5 \mathrm{~s}$ to $\mathrm{t}=$ 626 “injection end” plus 200-500 s.

627

628 The operator-set boundaries for the distributions were uniformly set to limit $K_{\mathrm{d}}$ values in the 629 interval from $10^{-18}$ to $10^{-6} \mathrm{M}$, and $k_{\text {off }}$ values in the interval from $10^{-7}$ to $10^{-2} \mathrm{~s}^{-1}$ to ensure 630 comparable and best quality fits reflected in a high signal to rmsd ratio.

631 The distribution $P\left(k_{\mathrm{a}}, K_{\mathrm{A}}\right)$ is calculated using the discretization of the equation:

$$
R_{\text {total }}=\int_{K_{A \min }}^{K_{A \max }} \int_{k_{a \min }}^{k_{a \max }} R\left(k_{a}, K_{A}, C_{\text {analyte }}, t\right) P\left(k_{a}, K_{A}\right) d k_{a} d K_{A}
$$

633 in a logarithmic grid of $\left(k_{\mathrm{a}}, i, K_{\mathrm{A}}, i\right)$ values with 15 and 18 grid points distributed on each axis 634 respectively. This was done through a global fit to association and dissociation traces at the above635 mentioned analyte concentrations. Tikhonov regularization was used as described by Zhao et al.

$636{ }^{57}$ at a confidence level of $P=0.95$ to determine the most parsimonious distribution that is 637 consistent with the data, showing only features that are essential to fit the data.

638 Flow-induced dispersion analysis (FIDA)

639 Flow Induced Dispersion Analysis (FIDA) experiments were performed using a FIDA 1 640 instrument employing light-emitting-diode (LED) induced fluorescence detection using an 641 excitation wavelength of $480 \mathrm{~nm}$ and emission wavelength $>515 \mathrm{~nm}$ (Fida Biosystems ApS, 642 Copenhagen, Denmark). Non-coated capillaries with inner diameter $75 \mu \mathrm{m}$, outer diameter 375 
$643 \mu \mathrm{m}$, total length $100 \mathrm{~cm}$, and length to detection window $84 \mathrm{~cm}$ (Fida Biosystems) were applied.

644 Indicator samples composed of 50 or $20 \mathrm{nM}$ Atto488-labeled HJs carrying 0-3 NPs or NIPs in

645

646

647

648

649

650

651

652

653

654

655

656

657

658

659

660

661

662

663

664

665

666

667

668

669

670

671 assay buffer (Hanks Balanced Salt Solution supplemented with 0.1\% BSA, 5\% FCS or anti-NP mAbs (Abcam; ab206523)) were analyzed using the following procedure. Initially, the capillary was flushed at $3500 \mathrm{mbar}$ for $120 \mathrm{~s}$. Then, assay buffer was applied at $1500 \mathrm{mbar}$ for $20 \mathrm{~s}$, followed by the indicator sample at $50 \mathrm{mbar}$ for $10 \mathrm{~s}$, and finally, assay buffer at $400 \mathrm{mbar}$ for $180 \mathrm{~s}$. The Taylorgrams were interpreted using the FIDA software suite, version 2.04 (Fida Biosystems ApS, Copenhagen, Denmark) and a Taylorgram fraction setting of $75 \%$, providing the apparent hydrodynamic radius of Atto488-labeled HJs (indicator).

\section{Equilibrium calculations for monovalent antigens}

We employed a simple model considering individual $\mathrm{Fab}$ arms as independent binding sites and 1:1 Ag binding. Considering that total $\mathrm{Ag}=$ freeAg $+\mathrm{FabAg}$ and totalFab $=$ freeFab $+\mathrm{FabAg}$, we can substitute in the expressions of freeAg and freeFab in the equation for the binding constant $\mathrm{K}_{\mathrm{D}}$ $=$ freeAg $\times$ freeFab $/$ FabAg, to achieve:

9

$$
K d=\frac{(\text { totalAg }- \text { FabAg }) *(\text { totalFab }- \text { FabAg })}{F a b A g}
$$

Abbreviating totalAg to tAg and totalFab to tFab for simplicity, and solving for FabAg/tFab, we arrive at:

64

$$
\text { Eq1: Fraction bound }=\frac{F a b A g}{t F a b}=\frac{t A g+t F a b+K d \pm \sqrt{(t A g+t F a b+K d)^{2}-4 * t A g * t F a b}}{2 * t F a b}
$$

Which is equivalent to the well-established equilibrium binding equation as given in ${ }^{58}$.

We generated an R script based on this equation with the assumption that B cells on average carry approximately 100,000 BCRs, equivalent to 200,000 Fab arms, and using standard conditions under which we had $10^{6} \mathrm{~B}$ cells pr. $\mathrm{ml}$ in $200 \mu \mathrm{l}$ in-well volume, for a total of $200,000 \mathrm{~B}$ cells. This is equivalent to a total Fab arm concentration of: 


$$
[\text { Fab }]=\frac{200,000 \mathrm{Fab} \text { per } B \text { cell } * 200,000 \mathrm{~B} \text { cells }}{200 * 10^{-6} \mathrm{~L} * 6.022 * 10^{23} \text { molecules } / \mathrm{mol}}=\frac{4 * 10^{10}}{12 * 10^{19}} M=0.33 \mathrm{nM}
$$

674

675 However, depending on the model, B1-8i or B1-8hi, only approximately $12 \%$ or $50 \%$, 676 respectively, of B cells carry a receptor with specificity for hapten.

677

678

679

680

681

682

683

684

685

686

687

688

689

690

691

692

693

694

695

696

697

698

Hence, we can define a function:

$$
\text { Eq2: Fraction bound }=\text { function }(x, y, z)\left(\frac{x+y * a+z-\sqrt{(x+y * a+z)^{2}-4 x * y * a}}{2 * y * a}\right)
$$

Where $x$ is the total concentration of antigen, $a$ equals the constant $3.320604 \times 10^{-10}$, which is modified by $y$, representing the fraction of cells carrying receptors specific for the antigen, and finally $z$ is the $K_{D}$ of the antigen-receptor pair being interrogated.

Plotting this across the 8 orders of magnitude from $10^{-11}$ to $10^{-3}$ for B1-8hi/NIP (freqB1-8 $=0.12$, $\left.\mathrm{K}_{\mathrm{D}}=1.9 \times 10^{-9}\right), \mathrm{B} 1-8 \mathrm{hi} / \mathrm{NP}\left(\right.$ freqB1-8 $\left.=0.12, \mathrm{~K}_{\mathrm{D}}=7.6 \times 10^{-8}\right), \mathrm{B} 1-8 \mathrm{i} / \mathrm{NIP}\left(\right.$ freqB $\left.1-8=0.5, \mathrm{~K}_{\mathrm{D}}=1 \times 10^{-7}\right)$, B1-i/NP (freqB1-8 $=0.5, K_{D}=4 \times 10^{-6}$ ), yields the binding curves shown in Figure S10.

We generated a vector of 80,000 values, distributed with 10,000 random values across each of the 8 logarithmic intervals from $10^{-11}$ to $10^{-3}$. We then calculated the fraction bound for the numbers in this vector using each of the binding curves, then calculated the mean, and finally the root mean square error (RMSE), using the formula:

$$
R M S E=\sqrt{\frac{\sum_{i=1}^{n}(f 1(i)-f 2(i))^{2}}{n}}
$$

Where $f 1$ and $f 2$ refer to Eq2 with any specified antigen/receptor pairs and corresponding values of $y$ and $z$. Example data for one randomized vector is shown in Table S1. Taken together, this 
revealed that B1-8hi:NP and B1-8i:NIP were very similar in their binding profiles, with a root mean square error of only $4.84 \%$ of the mean.

To evaluate the robustness of our analyses, we asked what impact a $10 \%$ or $20 \%$ deviation in the number of B1-8 cells would have on calculated binding equilibria across the randomized vector. The results are shown in Table S2. The relatively small RMSE resulting from even 20\% deviations in the number of B1-8 cells indicated that slight experimental variations would not significantly perturb the examined activities under the given conditions.

\section{Mice}

The B1-8hi line ${ }^{24}$ was kindly provided by Thomas Winkler, and the B1-8i line ${ }^{30}$ was kindly provided by Anja Hauser. The mice were maintained in our SPF vivarium at the Department of Biomedicine, Aarhus University, in IVCs on a standard 12-hr light/dark cycle, with standard chow and water ad libitum.

\section{Magnetic-activated cell sorting (MACS)}

MACS cell separation was performed using a modified version of official Miltenyi Biotec MACS protocol. Lymphoid tissue was harvested from euthanized mice and processed through $70 \mu \mathrm{m}$ cell strainers using MACS buffer (Dulbecco's Phosphate Buffered Saline (DPBS), 2\% Heat Inactivated Fetal Bovine Serum (FBS), 2 mM EDTA) and RBC Lysis buffer afterwards. Lysed cells were filtered again through $70 \mu \mathrm{m}$ cell strainer and obtained PBMCs were treated with Fc Block (Purified Rat Anti-Mouse CD16/CD32, Clone 2.4G2) for $5 \mathrm{~min}$, followed by Miltenyi Biotec Biotin Ab-cocktail for 30 min. Following that, cells were centrifuged at $200 \mathrm{G}, 4^{\circ} \mathrm{C}, 10$ min, resuspended in $1 \mathrm{~mL}$ MACS and mixed with Miltenyi Biotec anti-biotin magnetic beads for 20 min. Cell suspension was then applied to Miltenyi Biotec LS column through $70 \mu \mathrm{m}$ cell strainer, after which the column was washed with additional $3 \mathrm{~mL}$ of MACS buffer. Both flow through (untouched B cells) and column eluate (remaining cells) were collected and analyzed using flow cytometry. 


\section{Flow Cytometry}

Relevant cells were obtained directly from lymphoid tissue or by thawing out frozen batches. Cells were diluted to $5 \times 10^{6}$ cells $/ \mathrm{mL}$ and $100 \mu \mathrm{L}$ used per well $\left(5 \times 10^{5}\right.$ cells/well $)$. Adjusted all-stain mixture was prepared initially, containing all dyes except for HJ variations. The dilutions of antibodies were as following: CD45R (Pacific Blue Rat Anti-Mouse CD45R (Clone RA3-6B2, BD)): 1/500, CD8a (PerCP/Cyanine5.5 anti-mouse CD8a (Clone 53-6.7, Biolegend)): 1/300, CD4 (PerCP anti-mouse CD4 (Clone RM4-5, Biolegend)): 1/300, IgMa (FITC Mouse Anti-Mouse IgM (a) (Clone DS-1, BD)): 1/500, IgMb (BV650 Mouse Anti-Mouse IgM (b) (Clone AF6-78, BD)): 1/300, Viability dye (Fixable Viability Dye eFluor 780 (eBioscience)): 1:2000. $144 \mu \mathrm{L}$ of all-stain mixture was mixed with $16 \mu \mathrm{L}$ of $\mathrm{HJ}-0 / 1 / 2 / 3 \mathrm{NP}, \mathrm{NIP}$ or NP-PE (NP (11)-PE (Phycoerythrin) (Biosearch Technologies), and titrations were made by taking $40 \mu \mathrm{L}$ of the previous dilution and adding to new $120 \mu \mathrm{L}$ of all-stain mix. $100 \mu \mathrm{L}$ of these adjusted all-stain mixtures were then added to $100 \mu \mathrm{L}$ of cells, and these were stained for 30 min at $4{ }^{\circ} \mathrm{C}$. Cells were washed twice in FACS buffer and analyzed within 2 hours on a NovoCyte Quanteon 4025 flow cytometer (Agilent, Santa Clara, CA) equipped with 4 lasers (405 nm, 488 nm, $561 \mathrm{~nm}$ and 640 $\mathrm{nm}$, and 25 fluorescence detectors). Data was acquired using NovoExpress software (v. 1.5.0, Agilent) and subsequent analysis was performed in FCSExpress v. 7 (De Novo Software, Pasadena, CA).

\section{Confocal Microscopy}

Frozen B1-8hi B cells were thawed out for imaging and diluted to $1 \times 10^{6}$ cells $/ \mathrm{mL}$ in thawing buffer (80\% Dulbecco's Phosphate Buffered Saline (DPBS), 20\% heat inactivated Fetal Bovine Serum (FBS (Merck))). Two-hundred microliters of cells were mixed with $200 \mu \mathrm{L}$ stain buffer (PBS, 10\% FCS, 0.1\% Sodium Azide) containing B220 (FITC Rat Anti-Mouse CD45R (Clone RA3-6B2, BD)) 1/500 dilution and HJs in described concentrations, for a final concentration of $5 \times 10^{5}$ cells/mL. Cells were stained for 20-30 min, washed twice with stain buffer and $200 \mu \mathrm{L}$ of cells were added to cytospin (Shandon Elliot). Cells were spun for $3 \mathrm{~min}$ at 800 RPM onto SuperFrost Plus Microscopy slides and washed twice with stain buffer. After washing for $5 \mathrm{~min}, 100 \mu \mathrm{L}$ of acetone was added to the slides in order to fix the cells. Cells were washed twice with stain buffer after fixation and mounted using SlowFade (SlowFade Diamond Antifade Mountant (Molecular 
Probes, Cat. No. S36967)). Imaging was performed using an Inverted Zeiss LSM710 laser scanning confocal microscope, using a 40x Plan-Neofluor (0.75 NA) objective. Images were analyzed with ImageJ.

\section{Calcium Flux using Flow Cytometry}

Cells for calcium flux were either freshly purified from lymphoid tissue or thawed out from frozen batches. Frozen cells were thawed out in B cell medium (BCM) (RPMI-1640 supplemented with 10\% FCS, $55 \mu \mathrm{M}$ 2-ME, 1\% P/S (100 Units/mL Penicillin, $100 \mu \mathrm{g} / \mathrm{mL}$ Streptomycin), $10 \mathrm{mM}$ HEPES, $1 \mathrm{mM}$ Sodium Pyruvate, and $1 \%$ MEM NEAA) warmed at $37^{\circ} \mathrm{C}$. Cells were diluted to $1 \times 10^{6}$ cells/mL in Hank's Balanced Slat Solution (HBSS) (Gibco, Cat. No. 14025050). A total of $3 \times 10^{6}$ cells in $3 \mathrm{~mL}$ were incubated with $3.5 \mu \mathrm{M}$ of Fluo-3 (AM) (Invitrogen, Cat. No. F1241) and $7.3 \mu \mathrm{M}$ of Fura Red (AM) (Invitrogen, Cat. No. F3021) for $30-45 \mathrm{~min}$ at $37{ }^{\circ} \mathrm{C}{ }^{59}$. After the incubation, the cells were washed once with HBSS and resuspended in $3 \mathrm{~mL}$ to a final concentration of $1 \times 10^{6}$ cells $/ \mathrm{mL}$. Of these, $240 \mu \mathrm{L}$ of cells were used per flux measurement and 30 $\mu \mathrm{L}$ were used to measure the background signal, before addition of stimulant. Goat Anti-Mouse IgG $(\mathrm{H}+\mathrm{L})$, Unconjugated, highly cross-adsorbed and biotinylated (Invitrogen, Cat. No. A16080) was used as a positive control for B1-8hi B cells, Purified Rat Anti-Mouse Ig $\lambda 1, \lambda 2 \& \lambda 3$ Light Chain (Clone R26-46 (RUO), BD Bioscience) was used a positive control for B1-8i B cells, HJ conjugates with $0,1,2$ or $3 \mathrm{NP} / \mathrm{NIP}$ conjugated in described concentrations were studied as activators. Either 170 or $130 \mu \mathrm{L}$ of cells were used to measure calcium flux after the addition of stimulant, at a flow rate of $35 \mu \mathrm{L} / \mathrm{min}$. Cells were analysed on a NovoCyte Quanteon 4025 (Agilent). Ratiometric analysis of Fluo-3 and Fura Red dyes was performed using FCS Express in order to determine changes in intracellular calcium levels.

\section{Imaging flow cytometry}

Freshly MACS-purified B cells were diluted to $4 \times 10^{6}$ cells $/ \mathrm{mL}$ and $500 \mu \mathrm{L}$ were used per sample. Each sample was firstly incubated with various configurations of HJ constructs containing 0- 3 NP/NIP in MACS buffer, for $30 \mathrm{~min}$. at $4^{\circ} \mathrm{C}, 10^{\circ} \mathrm{C}$ or $37^{\circ} \mathrm{C}$. Afterwards, the cells were stained with anti-B220 (FITC Rat Anti-Mouse CD45R (Clone RA3-6B2, BD)) and viability dye (Fixable Viability Dye eFluor 780 (eBioscience)) for $20 \mathrm{~min}$. at $4^{\circ} \mathrm{C}$ and Hoechst (Nordic Biosite, Cat. No. 
801

802

CDX-B0030-M025) for 10 min at RT. After staining, the cells were washed once and analyzed using an ImageStream ${ }^{\mathrm{X}}$ MkII (Amnis, Luminex Corporation) at x60 magnification. At least 5000 NP positive B cells were recorded for each sample. IDEAS software (v.6.2, Amnis, Luminex Corporation) was used for analysis.

\section{Primary nanobody structure}

The amino acid sequence for the anti-mouse kappa light chain nanobody (mKappa-Cys87) presented below was kindly provided by Dirk Görlich ${ }^{25}$. The complementarity determining regions (CDRs) are shown in red. For DNA-PAINT super-resolution microscopy, the serine residue at position 87 (Ser87) was replaced with a cysteine residue (Cys87) for site-specific labelling of a DNA oligonucleotide. This design was based on the study by Hansen et al. ${ }^{60}$, who obtained high conjugation efficiency with engineered cysteine residues slightly distant from the C-terminus.

\begin{tabular}{|c|c|c|c|c|c|}
\hline MGQVQLVES $\frac{10}{G}$ & GGWVQPGGSI & RLSCAASGFT & FSDTAMMWVR & QAPGKGREWV & AAIDTGGGYT \\
\hline YYADSVKGR $\frac{70}{F}$ & $\begin{array}{r}80 \\
\text { TISRDNAKNT }\end{array}$ & $\begin{array}{r}90 \\
\text { LYLOMNCLKP }\end{array}$ & $\begin{array}{r}100 \\
\text { EDTARYYCAK }\end{array}$ & $\begin{array}{r}110 \\
\text { TYSGNYYSNY }\end{array}$ & $\begin{array}{r}120 \\
\text { TVANYGTTGR }\end{array}$ \\
\hline 3 & 140 & & & & \\
\hline
\end{tabular}

\section{Nanobody expression and purification}

Plasmids encoding the DNA sequences for anti-murine kappa light chains with Ser-to-Cys mutation in position 87 were purchased from GenScript with a C-terminal 6xHis-tag in pET22b(+) expression vector carrying ampicillin (AMP) resistance. The Nbs were expressed in the E.coli strain BL21 (D3) (GenScript).

In brief, a single colony was transferred to $20 \mathrm{~mL} 2 \mathrm{xTY}$ medium supplemented with AMP at a final concentration of $100 \mu \mathrm{g} / \mathrm{mL}$ in a $100 \mathrm{~mL}$ conical flask and grown over night $(\mathrm{O} / \mathrm{N})(16 \mathrm{~h})$ at $37{ }^{\circ} \mathrm{C}, 220 \mathrm{RPM}$. The following day, the $\mathrm{O} / \mathrm{N}$ culture was transferred to a $5 \mathrm{~L}$ Erlenmeyer flask containing $2 \mathrm{~L}$ terrific broth (TB) medium supplemented with $200 \mathrm{~mL}$ salt buffer $\left(0.17 \mathrm{M} \mathrm{KH}_{2} \mathrm{PO}_{4}\right.$, $\left.0.72 \mathrm{M} \mathrm{K}_{2} \mathrm{HPO}_{4}, \mathrm{pH} 7\right), 2 \mathrm{~mL} \mathrm{MgCl}_{2}(2 \mathrm{M}), 20 \mathrm{~mL} \mathrm{10 \%}(\mathrm{w} / \mathrm{v})$ glucose solution and $2 \mathrm{~mL}$ AMP 
$823(100 \mathrm{mg} / \mathrm{mL})$. The culture was grown at $37^{\circ} \mathrm{C}$ and $120 \mathrm{RPM}$ to an optical density 600 (OD600)

8240.6 at which point the expression was induced with $0.5 \mathrm{mM}$ isopropyl $\beta$-D-1-thiogalactopyranoside 825 (IPTG) at $25{ }^{\circ} \mathrm{C} \mathrm{O} / \mathrm{N}$. The following day, cells were harvested and resuspended in lysis buffer 826 (1xPBS, $400 \mathrm{mM} \mathrm{NaCl}, 20 \mathrm{mM}$ imidazole) supplemented with $5 \mathrm{mM} \beta$-mercaptoethanol (BME) 827 prior to sonication for $5 \mathrm{~min}$ at 8 watts ( $20 \%$ amplitude) in pulses of $10 \mathrm{~s}$ and $10 \mathrm{~s}$ of rest. The cell 828 lysate was centrifuged twice at $8000 \mathrm{xg}$ for $20 \mathrm{~min}$ before the cleared supernatant containing the 830 $\mathrm{Nb}$ was collected and sterile filtered (first using a $0.45 \mu \mathrm{m}$ filter followed by a $0.22 \mu \mathrm{m}$ filter).

The $\mathrm{Nb}$ was purified using Äkta Start system (Cytiva) by loading the supernatant onto a HisTrap column (Cytiva) packed with Ni Sepharose High Performance affinity resin. Prior to elution, the column was washed thoroughly with binding buffer (1xPBS, $400 \mathrm{mM} \mathrm{NaCl}, 20 \mathrm{mM}$ imidazole, $\mathrm{pH} 7.4,5 \mathrm{mM} \mathrm{BME}$ ) to remove unspecific proteins. The $\mathrm{Nb}$ were eluted by running a linear gradient of binding buffer with elution buffer (1xPBS, $400 \mathrm{mM}, 500 \mathrm{mM}$ imidazole, $\mathrm{pH}$ 7.4). Fractions containing the $\mathrm{Nb}$ were collected, and using Amicon Ultra-4 Centrifugal Filter Units (3K cut off), the proteins were buffer-exchanged and concentrated in 1xPBS following the manufacturers' instructions.

\section{Nanobody conjugation of DNA-PAINT oligonucleotide}

842 (ThermoFisher) for $2 \mathrm{~h}$ on ice. Subsequently, the $\mathrm{Nb}$ was conjugated to the DNA-PAINT 843 oligonucleotide (7xR3 sequence: NB-5'-CTCTCTCTCTCTCTCTCTC-3') in a two-step reaction.

844 First, the C-terminus cysteine was reacted with 20-fold molar excess of the heterobifunctional 845 linker DBCO-PEG4-maleimide $\mathrm{O} / \mathrm{N}$ at $4{ }^{\circ} \mathrm{C}$ in a thiol-maleimide reaction. Excess linker was 846 removed using Zeba Spin Desalting columns (7K MWCO), and the sample was buffer-exchanged 847 to $1 x$ PBS (1 mM EDTA, pH 8). Subsequently, the DBCO-modified nanobody was reacted with 8485 -azide-modified DNA oligo in 1:5 molar ratio and incubated at $4{ }^{\circ} \mathrm{C} \mathrm{O} / \mathrm{N}$. The final nanobody849 DNA conjugate was purified by anion exchange chromatography on a Source Q 5/50 column (GE 850 Healthcare) using a linear gradient $(1 \mathrm{~mL} / \mathrm{min})$ with 1xPBS, pH 7.4 as binding buffer and 1xPBS $851+1 \mathrm{M} \mathrm{NaCl}, \mathrm{pH} 7.4$ as elution buffer.

\section{Immunofixation of B1-8hi B cells for DNA-PAINT}


MACS-purified B1-8hi B cells were fixed with pre-heated $\left(37^{\circ} \mathrm{C}\right) 4 \%$ paraformaldehyde (PFA) for $30 \mathrm{~min}$ at RT. After fixation cells were washed two times with 1xPBS and transferred to 6 channel $\mu$-Slide VI 0.5 glass bottom (Ibidi). Cells were immobilized on the glass surface by centrifugation at $1000 \mathrm{~g}$ for $15 \mathrm{~min}$ in a swinging bucket rotor using in-house built adaptors. Channels were rinsed with $1 x P B S$ to remove unbound cells and subsequently incubated with 0.1 $\mathrm{M} \mathrm{NH}_{4} \mathrm{Cl}_{2}$ solution for $5 \mathrm{~min}$ followed by $2 \mathrm{x}$ wash steps. Cells were permeabilized with $0.1 \%$ Triton X-100 (Sigma-Aldrich) for $5 \mathrm{~min}$ at RT followed by 2x wash. To assure cells remained immobilized to the glass surface, the slide was centrifuged once at $1000 \mathrm{~g}$ for $10 \mathrm{~min}$. Hereafter, cells were incubated with blocking buffer (1xPBS, 3\% BSA, 1 mM EDTA, 0.02\% Tween-20, and $0.05 \mathrm{mg} / \mathrm{mL}$ salmon sperm DNA) in the dark for $1 \mathrm{~h}$ at RT. The DNA-modified mKappa-Cys87 $\mathrm{Nb}$ was diluted to $30 \mathrm{nM}$ in blocking buffer and added to the cells for incubation at $4{ }^{\circ} \mathrm{C} \mathrm{O} / \mathrm{N}$. The following day, cells were washed $3 \mathrm{x}$ with $1 \mathrm{xPBS}$ and centrifuged at $1000 \mathrm{~g}$ for 10 min prior to post-fixation with pre-heated $\left(37^{\circ} \mathrm{C}\right) 4 \%$ PFA for 5 min at RT and followed by $2 \mathrm{x}$ wash.

\section{Microscope setup and DNA-PAINT image acquisition}

Fluorescence imaging was carried out on an inverted microscope (Nikon Instruments, Eclipse Ti2) with the Perfect Focus System, applying an objective-type TIRF configuration equipped with an oil-immersion objective (Nikon Instruments, Apo SR TIRF $\times 100$, NA 1.49, Oil). The $561 \mathrm{~nm}$ laser (MPB Communications, $2 \mathrm{~W}$, DPSS system) used for excitation was passed through a cleanup filter (Chroma Technology, ZET561/10) and coupled into the microscope objective using a beam splitter (Chroma Technology, ZT561rdc). Fluorescence was spectrally filtered with an emission filter (Chroma Technology, ET600/50m and ET5751p) and imaged using a sCMOS camera (Andor, Zyla 4.2 Plus) without further magnification, resulting in an effective pixel size of $130 \mathrm{~nm}$ (after $2 \times 2$ binning).

DNA-PAINT is a localization-based SR technique, in which the dye-labeled DNA-imager strands transiently bind to DNA docking strands that are stably attached to the molecule of interest. During the time of transient DNA-DNA binding, enough photons can be harvested at the same location to fit this diffraction-limited spot as a point source, while the freely diffusing imager strands are detected as background. For imaging, a field of view (FOV) with a size of $512 \times 512$ pixels was selected, and images were acquired using a laser power of $21 \mathrm{~mW}$ at the objective with 40,000 
884 frames and an integration time of $100 \mathrm{~ms} .80 \mathrm{pM}$ of Cy3b-labeled imager strands (5'-GAGAGAG885 3'-Cy3b) were used.

886

887

888

889

890

891

892

893

894

895

896

897

898

899

900

901

902

903

904

905

906

907

908 909

910

911

912

913

\section{Image analysis}

Image stacks were reconstructed using Picasso Localize ${ }^{61}$. Spots were detected based on a net gradient of 2000 and localizations were fitted to a Gaussian distribution using the least squares method. Drift correction was performed in two steps using Picasso Render first with redundancy cross-correlation (RCC) and second by picking at least 3 gold nano particles. Resulting images were filtered for high-precision localizations by rejecting localizations with fitted PSF sigma values $>1.4$-times the camera pixel size. After image reconstruction and filtering we obtained a Nearest Neighbor based Analysis $(\mathrm{NeNa})$ localization precision ${ }^{62}$ of $8.8 \pm 1.7 \mathrm{~nm}$.

\section{DBSCAN clustering analysis}

Representative parts covering the majority of the B cell surface were saved as a circular or rectangular region of interest of 4-11 $\mu \mathrm{m}^{2}$. Clusters were analyzed with the DBSCAN method ${ }^{26}$. DBSCAN identifies localization clouds by detecting a minimal number of localizations within a circle of a defined search radius. Based on the measured localization precision, we used a radius of $14 \mathrm{~nm}$ and a minimum number of localizations of 15 .

\section{Quantitative PAINT analysis}

To determine the number of molecules within one cluster, we made use of the programmable kinetics of DNA binding and unbinding and performed qPAINT. Influx rates $\xi$ were calibrated from at least 60 single binding sites $(n=1)$, picked per FOV. Dark times $\tau_{D}$ were determined by fitting a cumulative distribution function (CDF) individually for each SBS. The mean dark time $\tau_{D}^{*}$ was calculated for each FOV and used for influx rate calibration according to equation 1.

$$
n=\frac{1}{\xi \cdot \tau_{D}^{*}}
$$

From the measured $\tau_{D}^{*}$, we could calculate the SBS-on-rate according to equation 2 . We obtained good agreement of experimental and theoretical conditions when comparing the SBS on-rate in 
914 our images $\left(k_{o n}^{S B S}=(45 \pm 15) \cdot 10^{6} \frac{1}{M S}\right)$ to that of theoretical R3-kinetics from DNA origami 915 measurements $\left(k_{o n}^{t}=36 \pm 2.7 \cdot 10^{6} \frac{1}{M s}\right)$, demonstrating appropriate measures for SBS 916 calibration $^{63}$.

$$
\xi=k_{o n} \cdot c_{i}
$$

In the same way as for the SBS-calibration, every group of localizations identified by DBSCAN

921 cluster algorithm was assigned a dark time by CDF-fitting from which the number of molecules 922 could be estimated ${ }^{27}$.

924 In order to exclude traces with unspecific imager interactions, prior to SBS calibration as well as 925 qPAINT analysis, we implemented mean frame as well as standard deviation filters. These are 926 based on the theory that specific DNA-PAINT localizations are indicated by repetitive transient 927 binding events that are evenly distributed over the acquisition time, leading to a mean frame of 928 around half the number of total frames acquired. Unspecific binding is indicated by a 929 disproportional accumulation of binding events in a short amount of time, giving rise to a mean 930 frame shifted towards lower or higher frame numbers. To remove these localization clouds, we 931 fitted the mean frame value of all detected localization clouds, and the cut-off value was set at plus 932 or minus the standard deviation. Clustered events occurring around the range of the mean frame 933 cannot be removed by mean frame filtering, which is why we additionally applied a standard 934 deviation frame filter. As clustered events result in a mean frame located within the frames of their 935 random appearance, the resulting mean frame standard deviation is small, and therefore we 936 excluded all clusters with a mean frame standard deviation $<4000$ frames ${ }^{64,65}$. 


\section{Ethics statement}

Breeding and maintenance of transgenic animals and harvesting of cells for use in the described experiments were conducted in accordance with the guidelines of the European Community and was approved by the Danish Animal Experiments Inspectorate (protocol number 2017-15-020101319)

\section{Acknowledgments}

We are indebted to Simon Davis for insightful discussions and the first suggestion that the kinetic segregation model could be applicable to B cell activation. Anja Hauser and Thomas Winkler kindly provided the B1-8i Jk knock-out and B1-8hi models, respectively. Dirk Görlich kindly provided the anti-mouse-Kappa nanobody. We are grateful to Lisbeth Jensen and Thomas Wittenborn for expert technical assistance and Anne Færch Nielsen for critical review of our manuscript. We thank Charlotte Christie Petersen and Anja Bille Bohn for help with flow and imaging cytometry, and Christian Garm for assistance with imaging.

This study was mainly funded by the Danish National Research Foundation to the Centre for Cellular Signal Patterns (CellPAT) (DNRF135). SED was additionally funded by a Carlsberg Foundation Distinguished Fellowship (CF18-0446).

\section{Author Contributions}

SED and JK conceived and initiated the project. AF, MO, IB, JSN, RJ, JK and SED planned the experiments. TVJ and ST provided valuable feedback on the experimental approaches. AF, MO, IB, JSN, and DMD carried out the experiments. AF, MO, IB, JSN, RJ, PS, KJM, TVJ and SED analyzed data. AF, MO, IB and KJM prepared figures. AF, MO and SED wrote the first draft of the manuscript. All authors provided critical feedback on the manuscript and approved it in its final form.

\section{Competing Interests}


964 The authors declare that no competing interests exist.

Page 42 of 63 
A

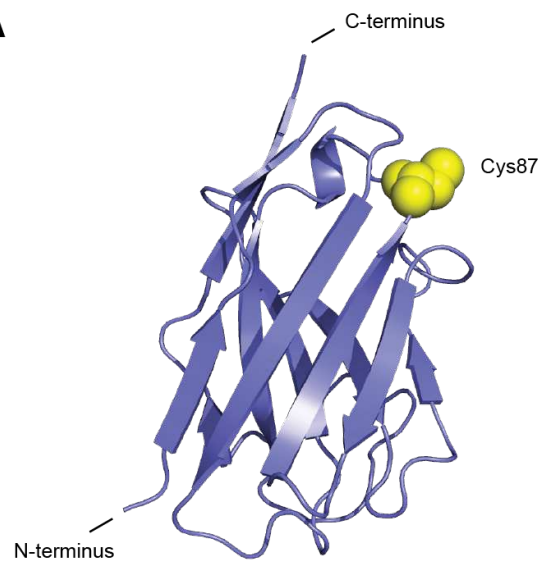

C

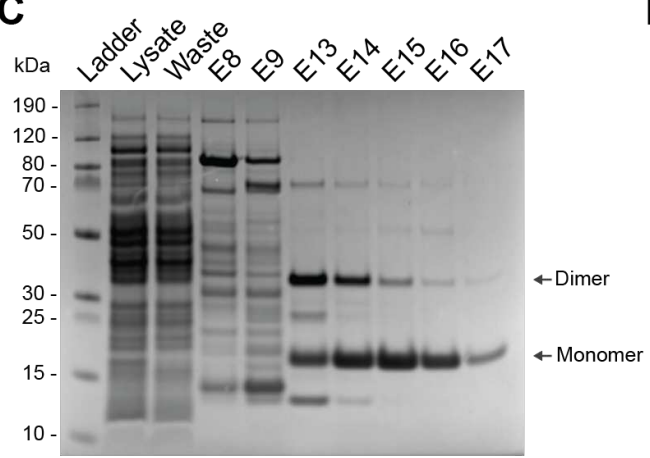

B

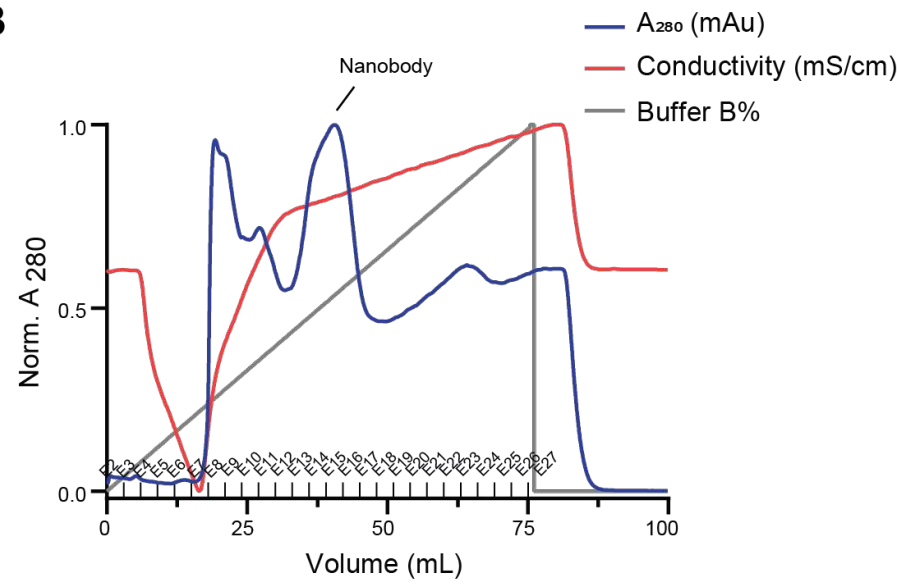

D

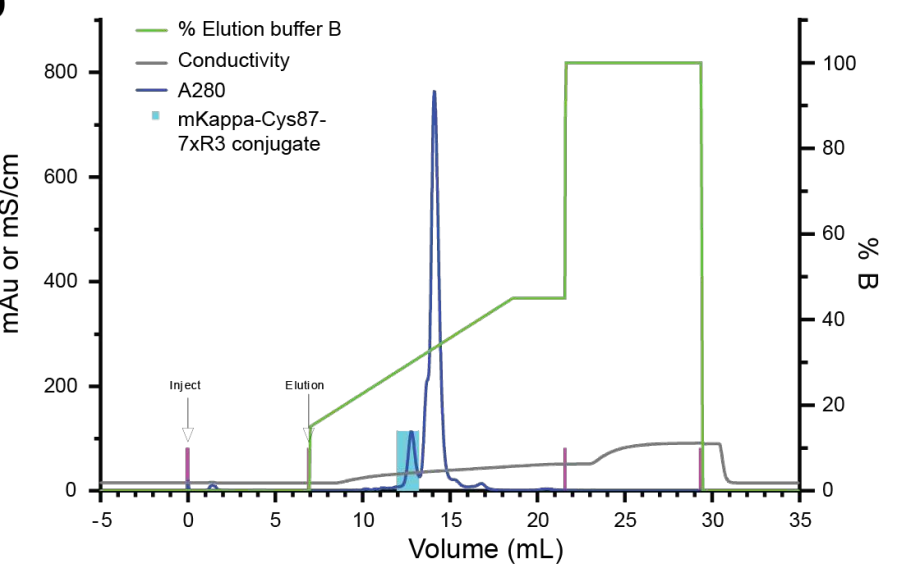

Figure S1. Nanobody expression and purification. (A) The anti-mouse kappa LC nanobody

968

969

970

971

972

973

974

975

976

977

978 (mKappa-Cys87) was expressed with a C-terminal cysteine residue by site-directed mutagenesis of Ser87 to Cys87 for subsequent site-specific conjugation. (B) Äkta FPLC chromatogram of nanobody elution profile. To avoid formation of disulphide bonds between the C-terminal free cysteine residues, the nanobody was eluted with $5 \mathrm{mM} \beta$-mercaptoethanol (BME) in the elution buffer. (C) SDS-PAGE gel (4-12\% Bis-tris) of the different nanobody fractions, from left: protein ladder, lysate, flow through (waste), eluted fractions E8-E17 corresponding to before and after nanobody peak. Despite the use of reducing agent, we still observed some dimer formation of the nanobody. Fractions E15-17 were pooled together for further work on the nanobody. The ladder is a PageRuler pre-stained protein ladder $(10-180 \mathrm{kDa})$. The gel was stained with Coomassie Brilliant blue. (D) FPLC chromatogram for the purification of the mKappa-Cys87-7xR3 conjugate used for subsequent imaging experiments. FPLC, fast protein liquid chromatography. 
A
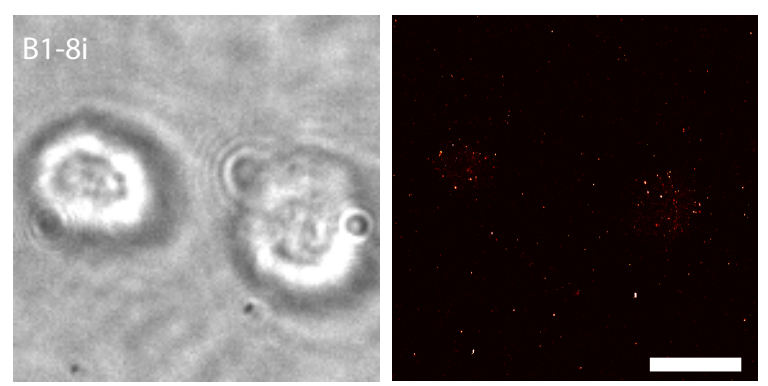

C
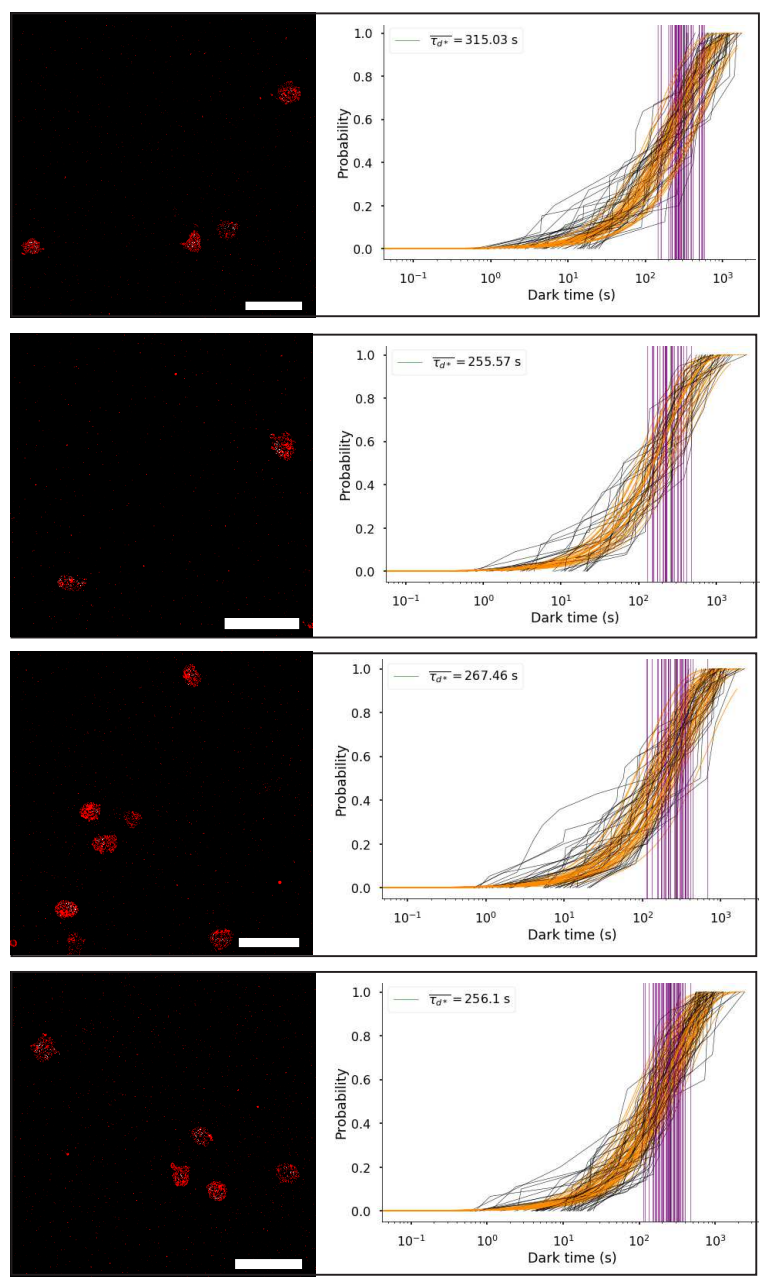

B
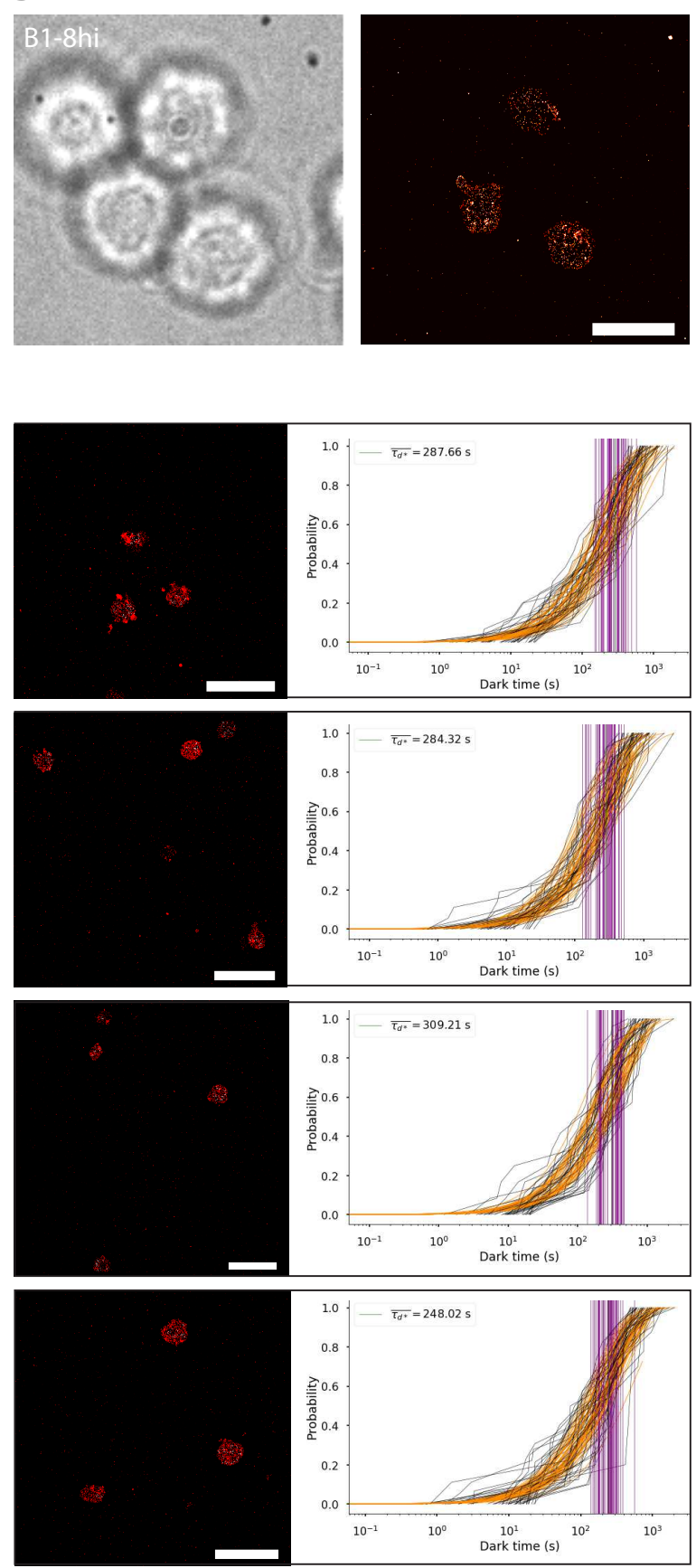

981 Figure S2. Dark time fits and filtering for DNA-PAINT imaging of B1-8hi B cells with anti-

982 kappa light chain nanobody. (A) Negative control for the anti-mouse kappa-light-chain

983 nanobody-7xR3. B1-8i cells are $>90 \%$ kappa-light chain-negative and show low signal. (B)

984 Positive control for the anti-mouse kappa-light chain nanobody-7xR3. B1-8hi B cells are $>80 \%$ 
985 kappa-light chain positive and show strong signal compared to the negative control. (C) Images 986 used for data analysis. Each box represents one of the 8 FOVs that were used for data analysis. 987 Left: FOV with 2-7 cells with DNA-PAINT data (red) and picked single binding sites (SBS, 988 white). Right: Traces of each picked SBS were analyzed and the dark times (black) were fitted as 989 a cumulative distribution function (orange). The mean over all individual dark times (violet) was 990 used as the dark time corresponding to SBSs (upper left box). Scale bars A and B: $5 \mu \mathrm{m}, \mathrm{C}: 10$ $991 \mu \mathrm{m}$. 
A

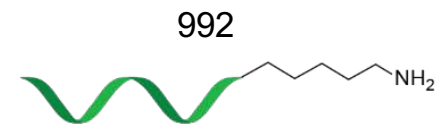

5' amino $Q$ oligo

994
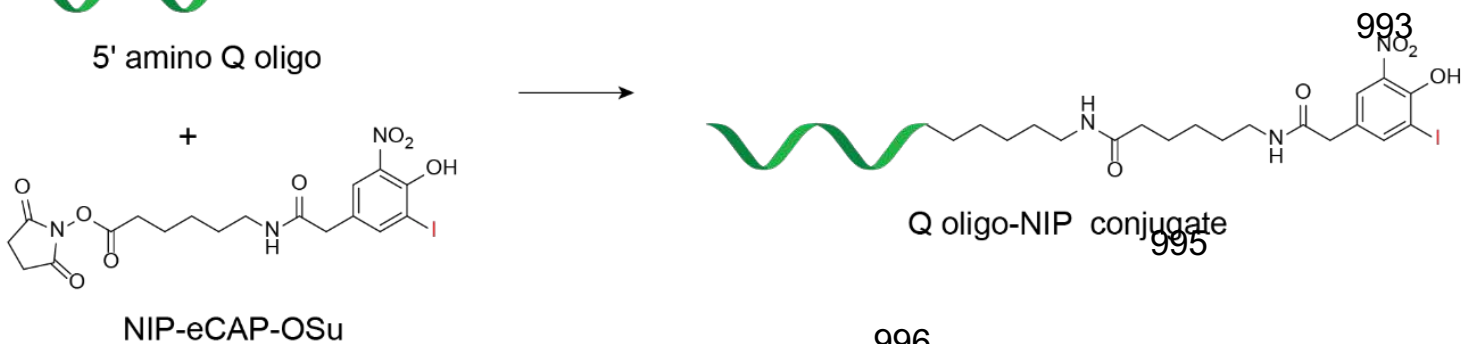

B

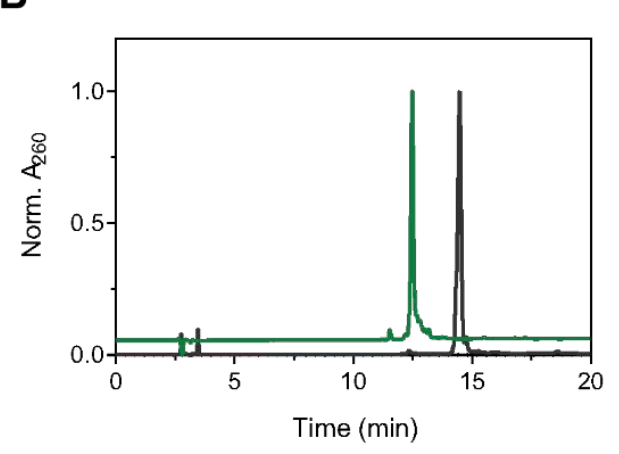

$-\mathrm{Q} 1$

- Q1-eCAP-NIP

D

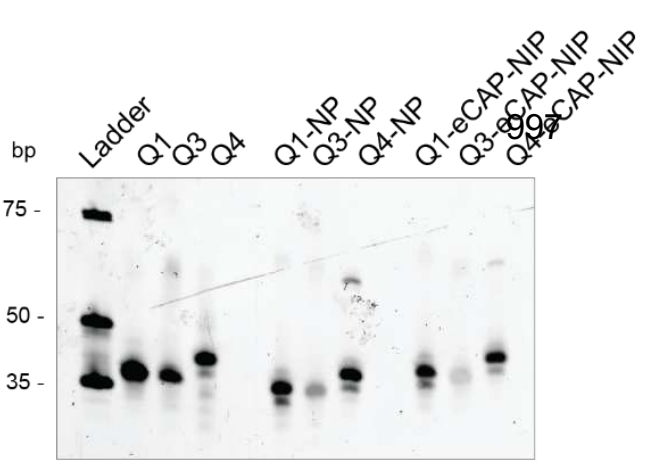

1002

1003

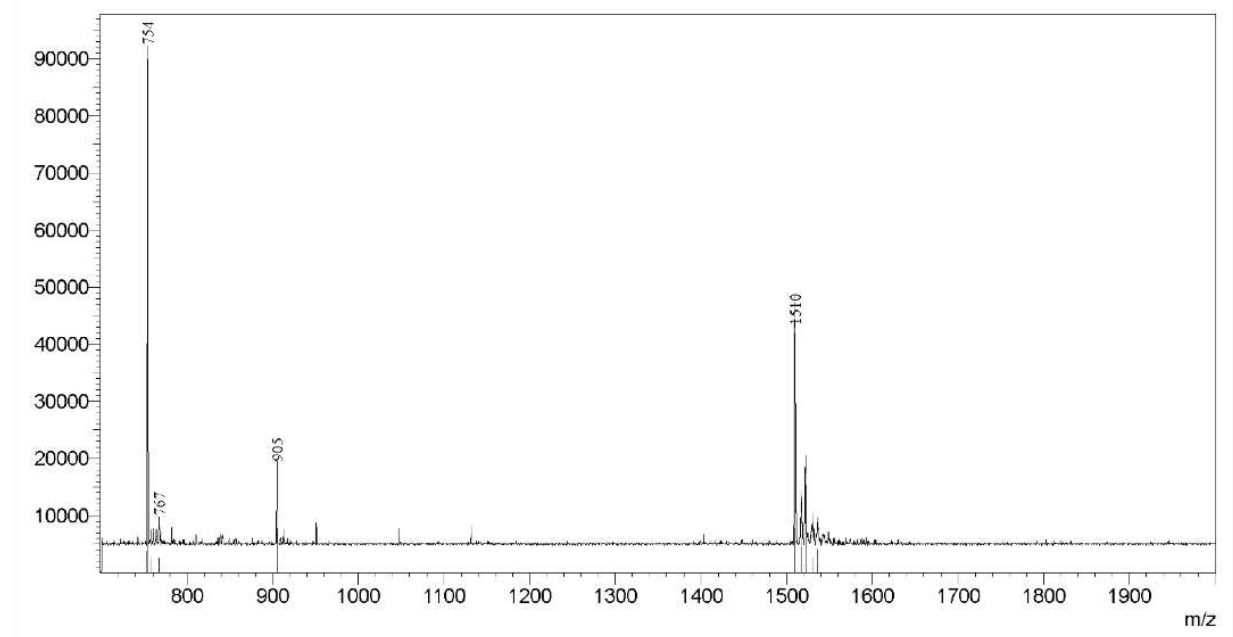

E

\begin{tabular}{|c|c|c|}
\hline Conjugate & Predicted $\mathbf{~ M W ~} \mathbf{( g / m o l})$ & Calculated $\mathbf{M W} \mathbf{~ g / m o l )}$ \\
\hline Q1-NP & 4292.90 & 4291.95 \\
\hline Q3-NP & 4316.93 & 4316.75 \\
\hline Q4-NP & 4411.99 & 4410.66 \\
\hline Q1-eCAP-NIP & 4531.96 & 4530.82 \\
\hline Q3-eCAP-NIP & 4555.98 & 4554.45 \\
\hline Q4-eCAP-NIP & 4651.04 & 4650.47 \\
\hline
\end{tabular}


1004 Figure S3. Functionalization Q oligos with NP or NIP haptens. (A) Reaction scheme showing 1005 the conjugation of NP hapten to the 5' amino-modified Q oligo using an activated NHS ester. (B) 1006 A representative RP-HPLC chromatogram of Q1-eCAP-NIP conjugate purification. (C) A 12\% 1007 denaturing PAGE gel indicating purified Q-NP and Q-eCAP-NIP (Q1-NIP) conjugates. (D) 1008 Representative LC-MS mass chromatogram of purified Q1-eCAP-NIP conjugate. The purity of 1009 the sample is indicated by the low background and high signal-to-noise ratio. (E) Table showing 1010 predicted and calculated masses of oligo-hapten (NP or NIP) conjugates. The predicted masses 1011 were determined from the chemical structure of the conjugates drawn in the software ChemDraw 1012 Professional 17.1 (PerkinElmer). Calculated masses were derived from LC-MS analysis of purified 1013 conjugates using the analysis method $\mathrm{H}^{+}$with a tolerance of $1 \mathrm{Da}$. These data are in accordance 1014 with the predicted mass values. NIP-eCAP-OSu: 4-hydroxy-3-iodo-5-nitrophenylacetyl-O1015 succinimide ester; NP: 4-hydroxy-3-nitrophenylacetyl; NIP: 4-hydroxy-3-iodo-51016 nitrophenylacetyl amino caproyl acetyl. 
A

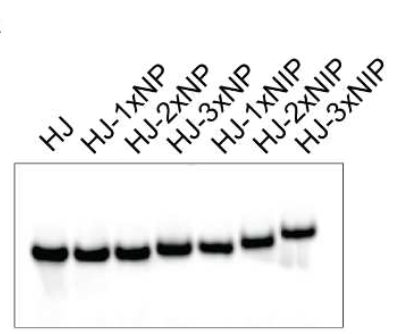

1021

1022

C

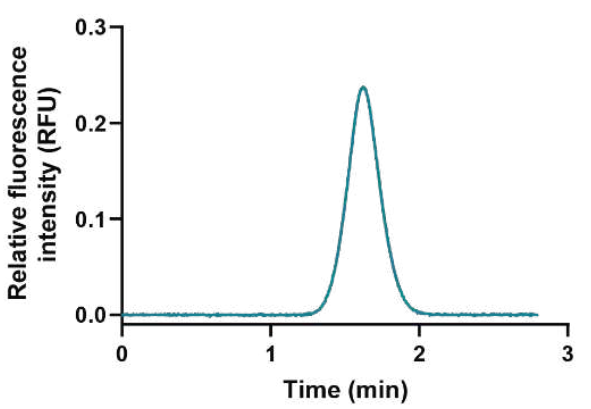

1027
B

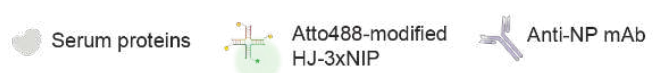
HJ-3XNIP
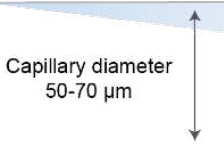

$+1$

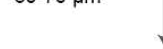

tit.

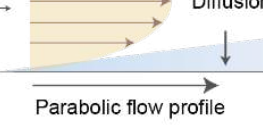

D
1018

$R_{\mathrm{H}} \sim 2.60 \mathrm{~nm}$
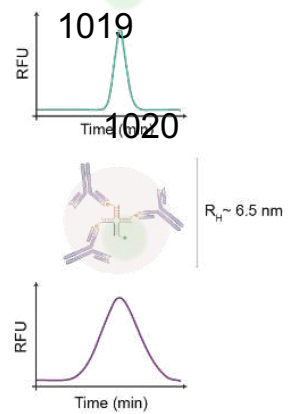

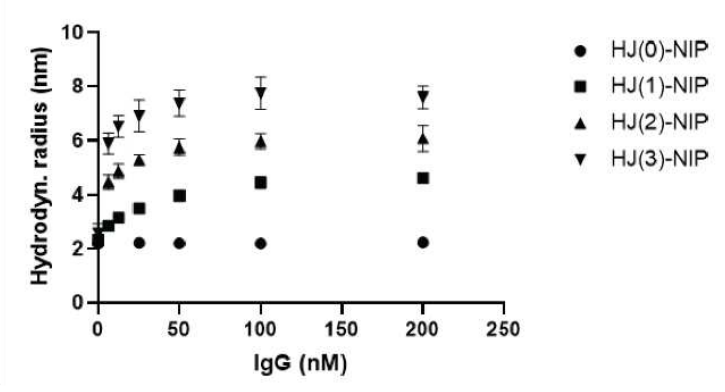

1028

1029

1030

1031

1032

1033

1034

1035

1036

1037

1038

1039

1040

1041

1042

Figure S4. Flow-induced dispersion analysis (FIDA) of HJ-NIP constructs. (A) A 12\% native PAGE gel of Atto488-modified HJs displaying 0-3 units of NP or NIP used in FIDA analysis. (B) Schematic illustration of the principle of FIDA technology. Figure modified from https://fidabio.com/technology. In brief, a dye-labelled indicator (Atto488-modified HJ-3xNIP) alone or in combination with an analyte (anti-NP $\mathrm{mAb}$ ) are injected into a capillary, where a parabolic flow profile of a buffer (typically PBS supplemented with BSA) is generated. In the capillary, the analyte is mixed with the indicator and as they diffuse toward the detector a Gaussian shaped signal, referred to as Taylorgram, appears. A complex formation between the analyte and indicator can be determined from the resultant signal compared to that of the indicator alone. As small molecules diffuse faster than larger ones, the shape of their signal will be more narrow than larger molecules or complexes. From the Taylorgrams, the apparent hydrodynamic radius $\left(\mathrm{R}_{H}\right)$ as well as dissociation constants $\left(\mathrm{K}_{\mathrm{D}}\right)$ of individual molecules and complexes can be derived ${ }^{32,33}$. (C) A representative Taylorgram of Atto488-modfiied HJ-1xNP in Hanks Balanced Salt Solution (HBSS) supplemented with 0.1\% BSA. (D) Apparent hydrodynamic radii of indicated AF488labelled HJs $(20 \mathrm{nM})$ as a function of an increasing anti-NP mAb concentration $(0-200 \mathrm{nM})$ 
1043 analyzed by FIDA in HBSS containing 0.1\% BSA at pH 7.4. Each sample was pre-incubated for $104430 \mathrm{~min}$ at RT ( $\mathrm{n}=3$, error bars indicate standard deviation). As seen, the apparent size of naked 1045 HJ remain the same at increasing anti-NP mAb concentration, however HJs displaying 1-3 NIPs 1046 increase gradually in size corresponding to the number of mAbs each HJ-NIP construct can bind. 1047 For all three HJ-NIP variants, a plateau is reached at $100 \mathrm{nM}$ anti-NP mAb, which indicates that 1048 no immune complex oligomerization (aggregate formation) is taking place. Thus, HJ-3xNIP can 1049 bind at most three mAbs, HJ-2xNIP two mAbs and HJ-1xNIP one mAb. 
A

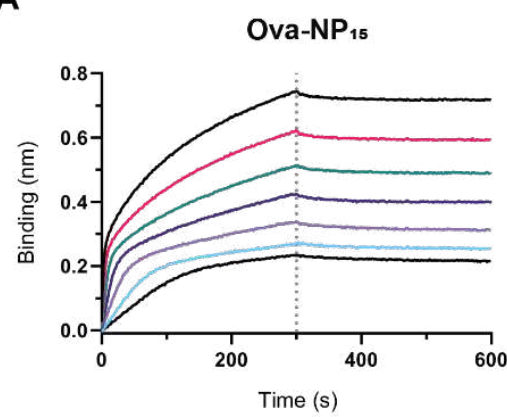

D

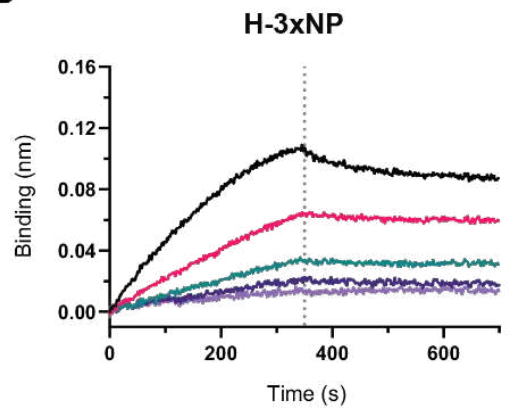

B

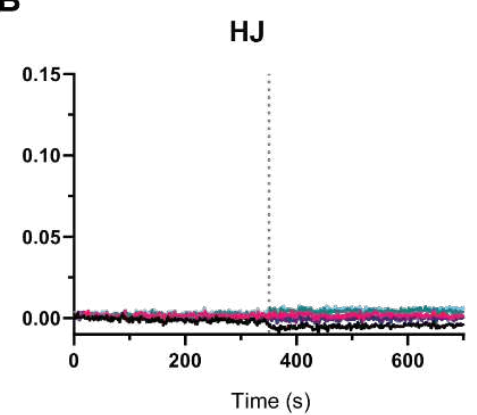

E

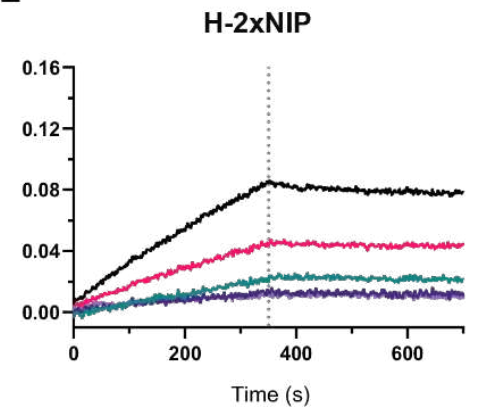

C

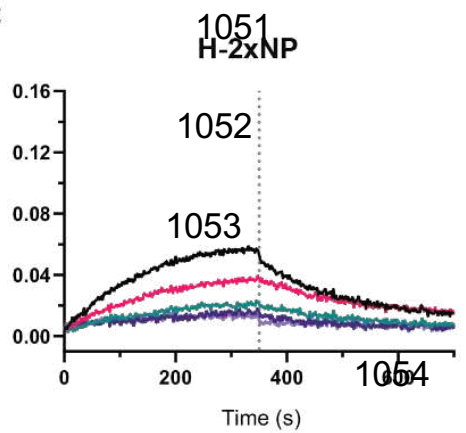

$\mathbf{F}$
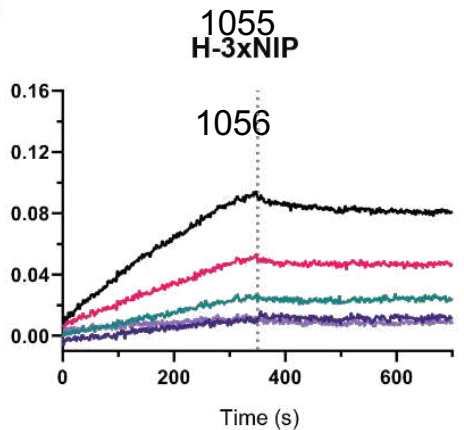

1058 Figure S5. Binding profile of haptenated HJs at low concentrations. (A) Ovalbumin modified 1059 with $15 \mathrm{NP}$ molecules $\left(\mathrm{Ova}^{-N_{15}}\right)(3.25-240 \mathrm{nM})$ was used as a positive control for our initial bio1060 layer interferometry binding assays to confirm our setup and the anti-NP mAb immobilized via 1061 protein A biosensors. (B) Binding profile of naked HJ used as a negative control (0.313-20 nM). 1062 (C-D) Sensorgrams of HJ-2xNP or $-3 x N P$ in the low concentration range $(0.125-2 \mathrm{nM})$. (E-F) 1063 Binding profiles of HJ-2xNIP and -3xNIP (0.063-1 nM). For all samples, the used concentration 1064 of anti-NP mAb, immobilized on the protein A sensors, was $1 \mu \mathrm{g} / \mathrm{mL}$ in a total volume of $200 \mu \mathrm{L}$. 1065 All samples were diluted in binding buffer in a 2-fold serial dilution in the described concentration 1066 intervals. 
A

HJ-1xNP
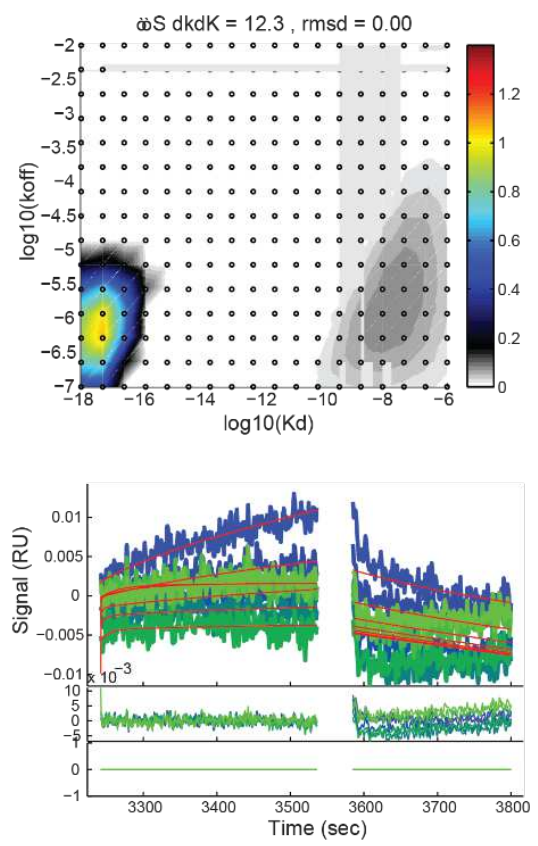

B

\section{HJ-1xNIP}

$\ddot{\infty} \mathrm{S} \mathrm{dkdK}=11.9$, rmsd $=0.00$
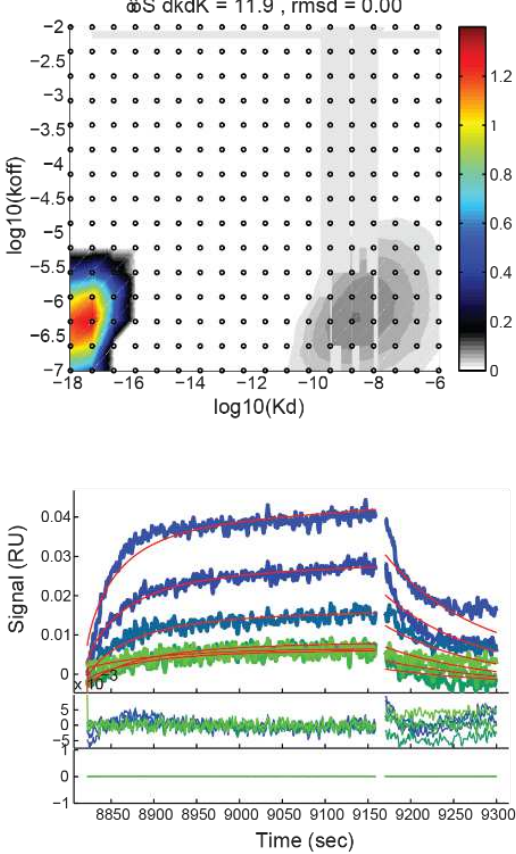

Signal Max 1.4

HJ-2xNP
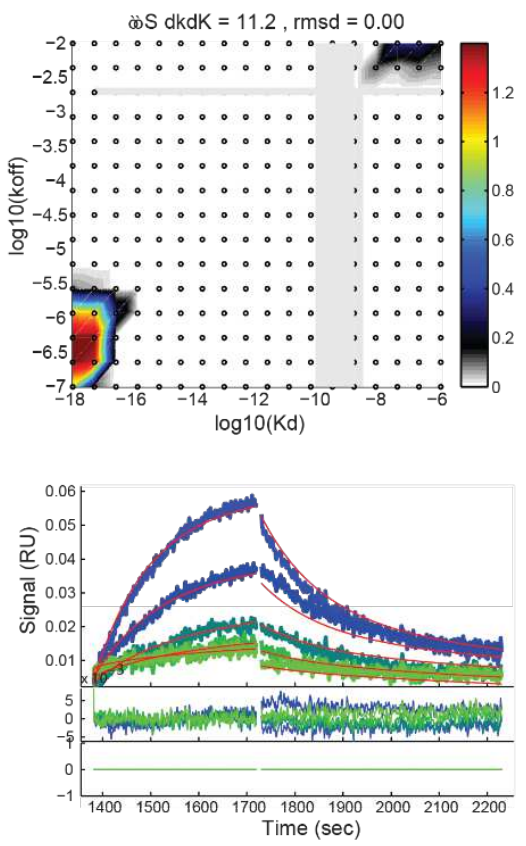

Signal Max 1.4

\section{HJ-2xNIP}

क̈S dkdK $=11.9$, rmsd $=0.00$
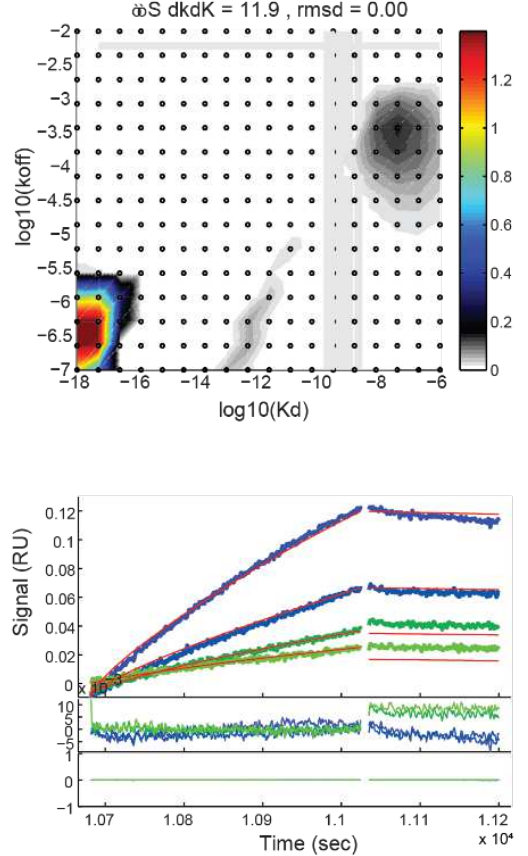

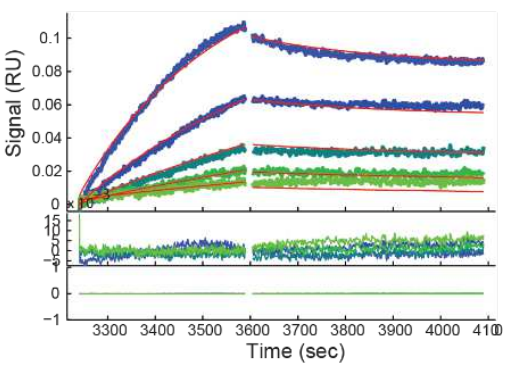

1070

HJ-3xNP

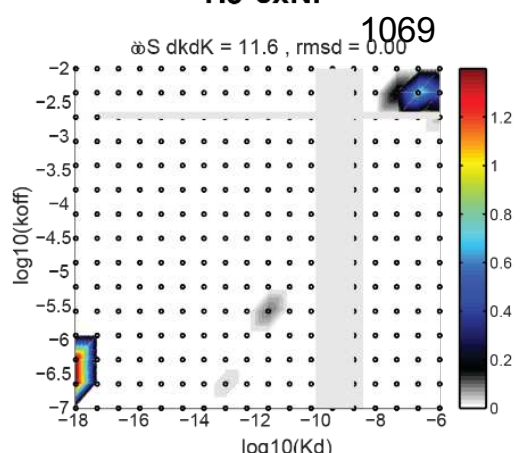

$\log 10(\mathrm{Kd})$

\section{HJ-3xNIP}
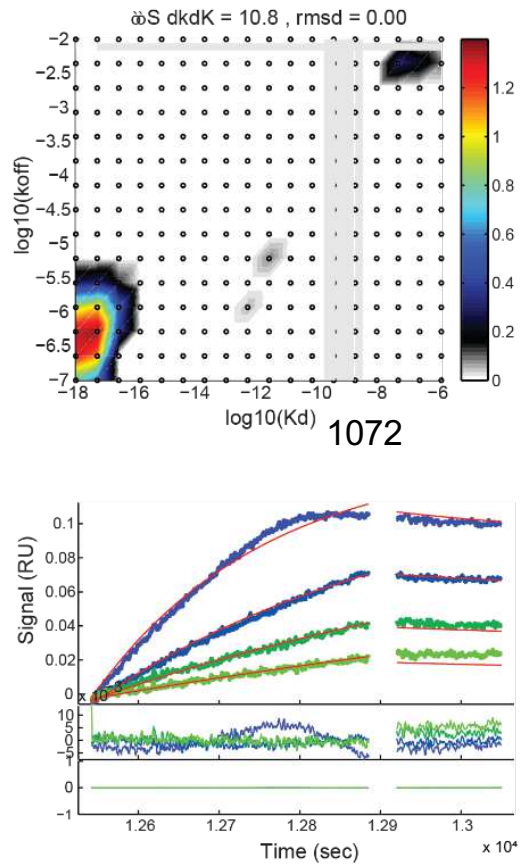
1073 Figure S6. EVILFIT analysis on HJ binding profile. (A) EVILFIT analysis of HJ-NP constructs 1074 based on following concentrations; $\operatorname{HJ}-1 \mathrm{xNP}(20,10,5,2.5,1.25,0.625,0.313 \mathrm{nM}), \mathrm{HJ}-2 \mathrm{xNP}$ and 1075 HJ-3xNP $(5,2.5,1.25,0.625,0.313 \mathrm{nM})$. Upper panels show the unmagnified (signal max 1.4) 2D 1076 plots for individual HJ-NP. At this scale, only for HJ-3xNP two small populations in the low $\mathrm{K}_{\mathrm{D}}$ 1077 and slow off-rate $\left(\mathrm{K}_{\mathrm{off}}\right)$ region are observed. Lower panels represent raw binding data and curve 1078 fits (red) that follow data points nicely as shown by residual plots below. (B) Upper panels indicate 1079 2D EVILFIT plots based on BLI data using the following concentrations; HJ-1xNIP (7, 3.5, 1.75, $10800.875,0.438,0.219 \mathrm{nM}), \mathrm{HJ}-2 \mathrm{xNIP}$ and HJ-3xNIP $(1.75,0.875,0.438,0.219 \mathrm{nM})$. Here, we see 1081 populations of low $\mathrm{K}_{\mathrm{D}}$ and slow $\mathrm{K}_{\text {off }}$ for both HJ-2xNIP and -3xNIP. Lower panels show curve 1082 fitting of the raw data. For all samples, concentrations that resulted in the best possible fit were 1083 chosen. 

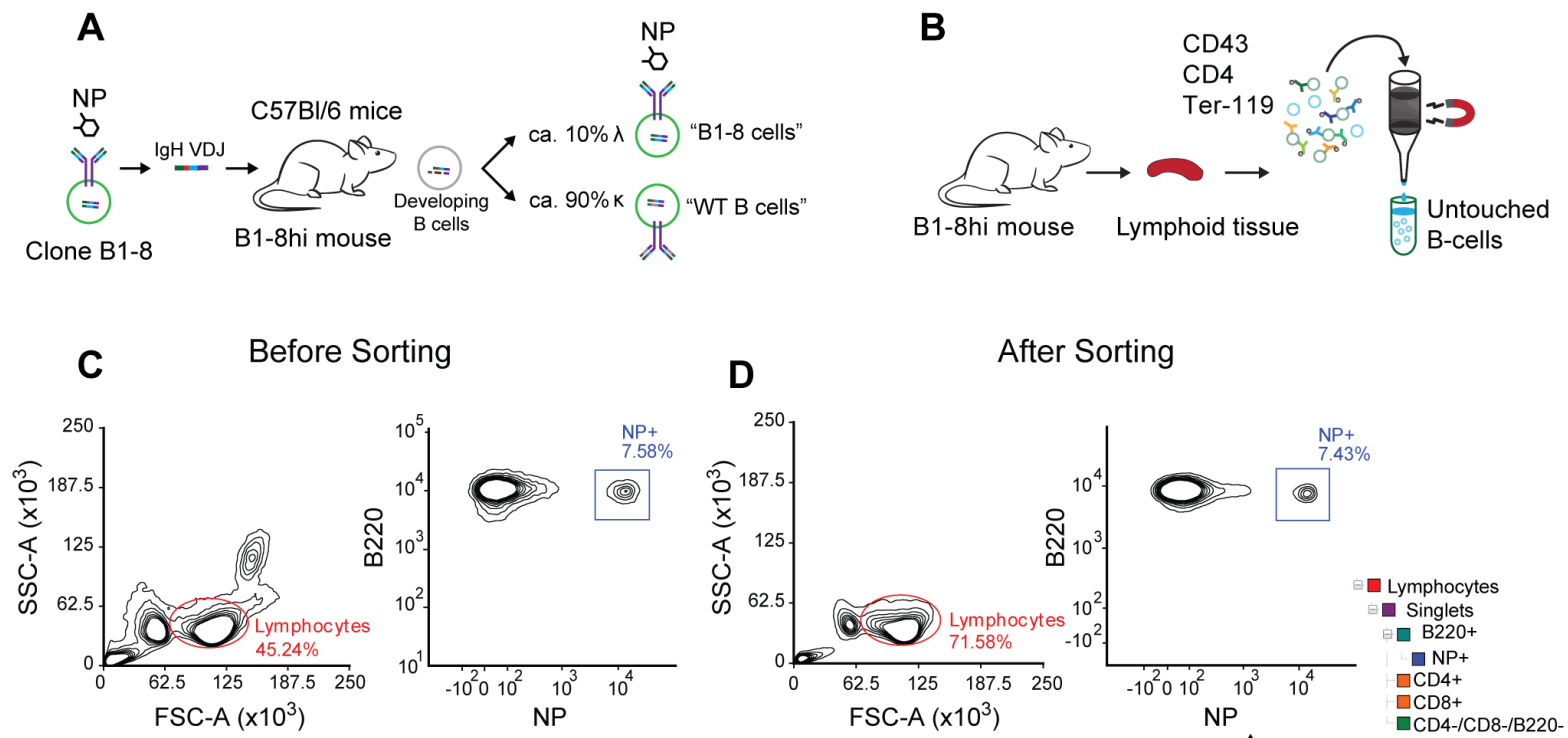

After Sorting
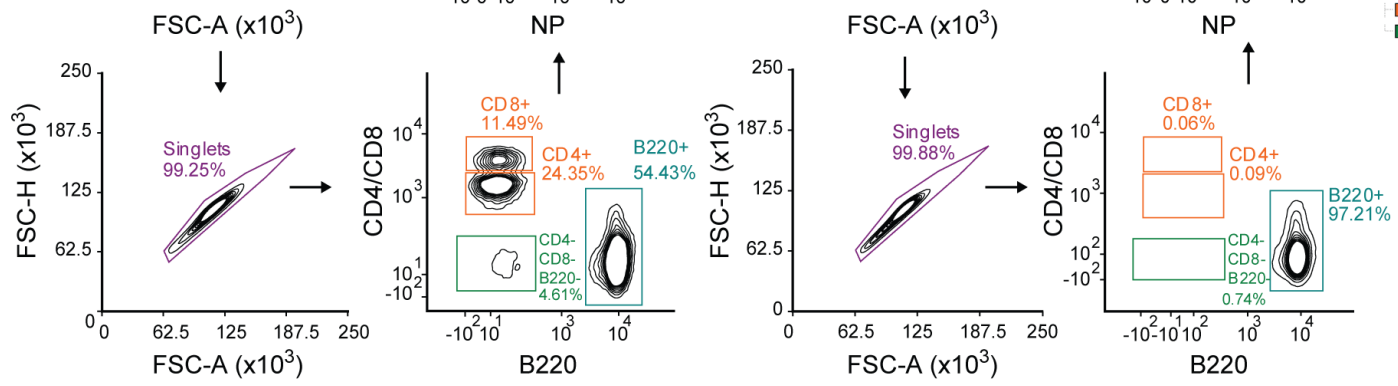
analysis by flow cytometry. (A) B1-8hi mouse model. Heavy chain V(D)J fragment from a B18hi B cell clone was introduced into $\mathrm{C} 57 \mathrm{Bl} / 6$ mice. The heavy chain of B cells developing in these mice will pair with either endogenous lambda $(\lambda)$ light chain or kappa $(\kappa)$ light chain. Only the $\sim 12 \%$ of B cells expressing both the introduced heavy chain and $\lambda$ light chain will be NP positive "B1-8hi B cells". (B) MACS purification of B1-8hi B cells. B cells were isolated using Magneticactivated cell sorting (MACS). Lymphoid tissue from B1-8hi mice was processed using MACS sorting protocol. All cells except B cells were marked with magnetic beads using MACS sorting cocktail, resulting in untouched B cells flowing through the column unretained. (C) Flow cytometric purity analysis of MACS isolated cells. B1-8hi derived B cells were analysed for purity, using flow cytometry, after MACS separation. For simplicity, the live-dead gating is not shown. The frequency of B cells increased from 54\% before sorting (left panels) to $97 \%$ after sorting (right panels). Isolated B cells retained the subpopulation of essential B1-8hi NP positive cells (NP+). 


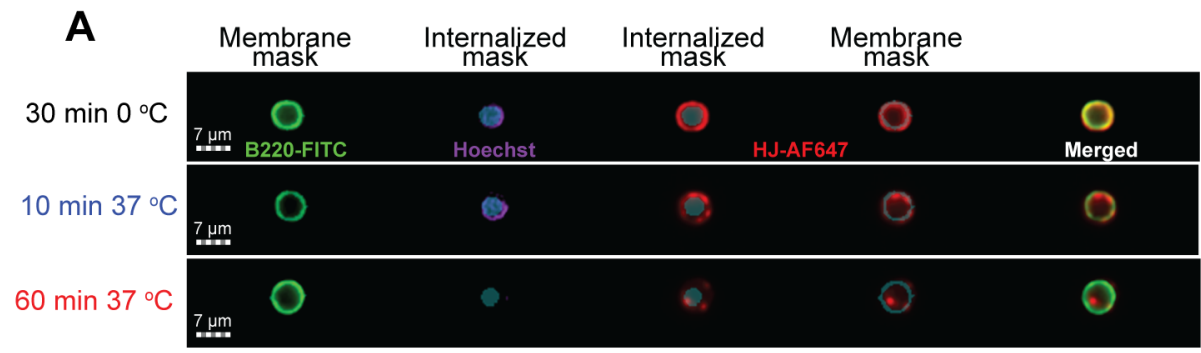

B

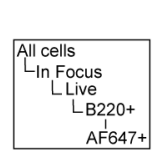

$1: 1$
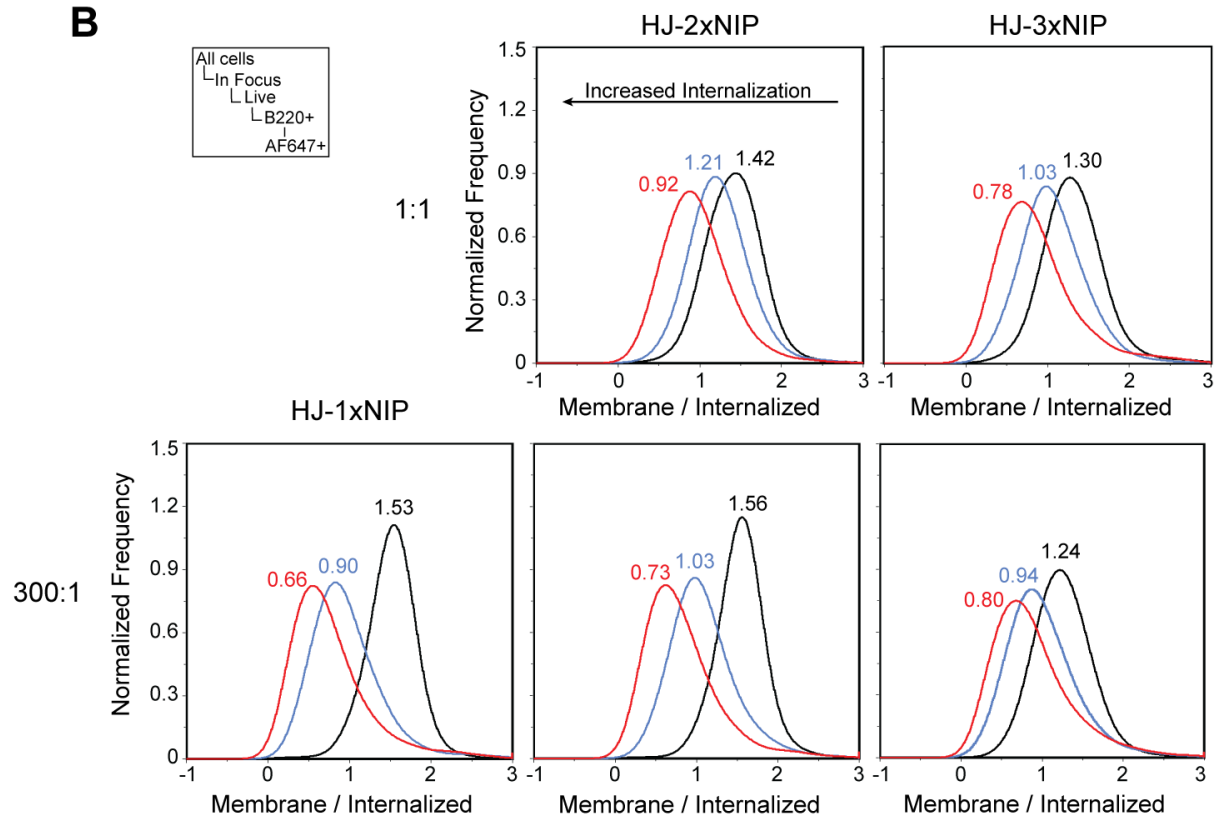

1103 Figure S8. Uptake and internalization of HJs by B1-8hi B cells measured by imaging flow cytometry. (A) As also shown in main Figure 4, a masking method was used to measure the amount of HJ signal (red) on the surface of the cells and within the cells. Membrane and 1106 internalization masks were made based on the B220 marker (green), either overlapping with it or excluding it. Nuclear Hoechst stain (blue) shows the potential overlap of internalized mask with

1108 the nucleus. (B) AF647 signal from different HJ constructs within these masks was quantified and 1109 plotted as membrane/internalized ratio for different valencies, stoichiometries, as well as 1110 incubation conditions (time and temperature). The curves represent between 3,000 and 5,000 1111 individual cells, and the median is given above each curve. 


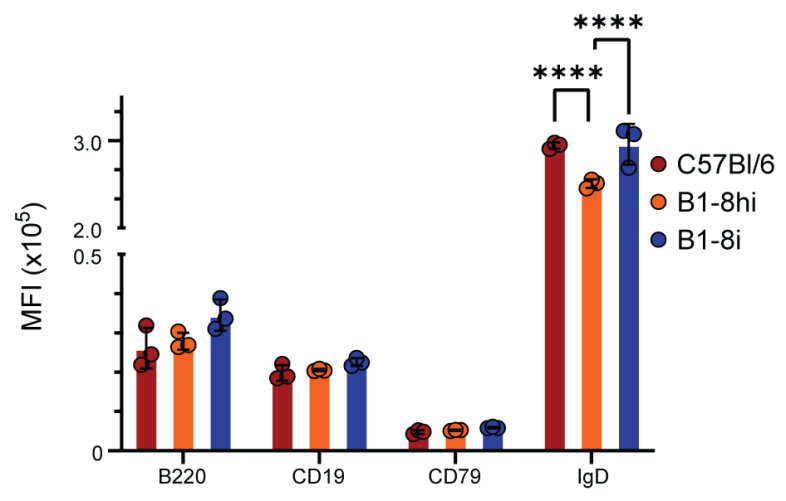

1116 Figure S9. Flow cytometric evaluation of surface expression of BCR constituents and 1117 associated molecules in C57BI/6, B1-8hi and B1-8i models. Median fluorescence intensities for 1118 B220, CD19, CD79 and IgD were evaluated upon flow cytometric analysis of splenocytes from $1119 \mathrm{C} 57 \mathrm{Bl} / 6, \mathrm{~B} 1-8 \mathrm{hi}$ and B1-8i models. Mean \pm SD from 3 individual mice per group. Statistical 1120 analysis was performed using two-way ANOVA with Tukey’s post-test using alpha $=0.05 ; * * * *$ $1121=\mathrm{p}<0.001$. 


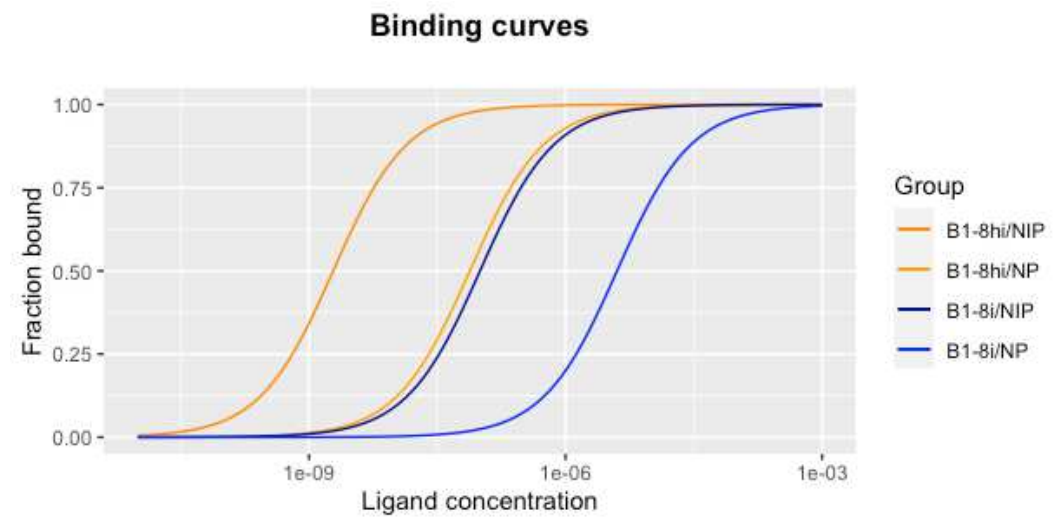

Figure S10. Calculated binding curves for B1-8hi/NIP, B1-8hi/NP, B1-8i/NIP, and B1-8i/NP.

1126 Binding curves were based on $e q 2$, with $x$ as the total concentration of antigen, $a$ equal to the 1127 constant $3.320604 \times 10^{-10}$, which is modified by $y$, representing the fraction of cells carrying 1128 receptors specific for the antigen, and finally $z$ is the $\mathrm{K}_{\mathrm{D}}$ of the antigen-receptor pair being 1129 interrogated. This was plotted in R across 8 orders of magnitude from $10^{-11}$ to $10^{-3}$ for B1-8hi/NIP $1130\left(\right.$ freqB1-8 $\left.=0.12, \mathrm{~K}_{\mathrm{D}}=1.9 \times 10^{-9}\right), \mathrm{B} 1-8 \mathrm{hi} / \mathrm{NP}\left(\right.$ freqB1-8=0.12, $\left.\mathrm{K}_{\mathrm{D}}=7.6 \times 10^{-8}\right)$, B1-8i/NIP (freqB1$\left.11318=0.5, \mathrm{~K}_{\mathrm{D}}=1 \times 10^{-7}\right)$, and B1-i/NP $\left(\right.$ freqB $\left.1-8=0.5, \mathrm{~K}_{\mathrm{D}}=4 \times 10^{-6}\right)$. 


\begin{tabular}{|c|c|c|c|c|c|}
\hline \multirow{2}{*}{} & \multirow{2}{*}{ mean } & \multicolumn{4}{|c|}{ RMSE } \\
\cline { 2 - 6 } & & B1-8hi:NIP & B1-8hi:NP & B1-8i:NIP & B1-8i:NP \\
\hline B1-8hi:NIP & 0.736 & - & 0.319 & 0.339 & 0.554 \\
\hline B1-8hi:NP & 0.537 & - & - & 0.026 & 0.339 \\
\hline B1-8i:NIP & 0.522 & - & - & - & 0.319 \\
\hline B1-8i:NP & 0.322 & - & - & - & - \\
\hline
\end{tabular}

1134

1135

1136

1137

1138

1139 the root mean square error (RMSE). 


\begin{tabular}{|c|c|c|c|c|c|}
\hline \multirow{2}{*}{} & \multirow{2}{*}{ Mean } & \multicolumn{4}{|c|}{ RMSE } \\
\cline { 3 - 6 } & & $+10 \%$ & $-10 \%$ & $+20 \%$ & $-20 \%$ \\
\hline B1-8hi:NIP & 0.736 & $1.08 \times 10^{-4}$ & $1.08 \times 10^{-4}$ & $2.16 \times 10^{-4}$ & $2.17 \times 10^{-4}$ \\
\hline B1-8hi:NP & 0.537 & $2.75 \times 10^{-6}$ & $2.75 \times 10^{-6}$ & $5.50 \times 10^{-6}$ & $5.50 \times 10^{-6}$ \\
\hline B1-8i:NIP & 0.522 & $8.80 \times 10^{-6}$ & $8.80 \times 10^{-6}$ & $1.76 \times 10^{-5}$ & $1.76 \times 10^{-5}$ \\
\hline B1-8i:NP & 0.322 & $2.12 \times 10^{-7}$ & $2.12 \times 10^{-7}$ & $4.23 \times 10^{-7}$ & $4.23 \times 10^{-7}$ \\
\hline
\end{tabular}

1142

1143 Table S2. Evaluation of robustness of analyses. Evaluation of the impact a $10 \%$ or $20 \%$ 1144 deviation in the number of B1-8 cells has on calculated binding equilibria across the vector of 114580,000 values, distributed with 10,000 random values across each of the 8 logarithmic intervals 1146 from $10^{-11}$ to $10^{-3}$. 


\section{References}

1148 1. Rajewsky, K. Clonal selection and learning in the antibody system. Nature 381, 751-758 (1996).

1149

1150

1151

1152

1153

1154

1155

1156

1157

1158

1159

1160

1161

1162

1163

1164

1165

1166

1167

1168

1169

1170

1171

1172

1173

1174

1175

1176

1177

1178

1179

1180

1181

1182

1183

1184

1185

1186

1187
2. Akkaya, M., Kwak, K. \& Pierce, S.K. B cell memory: building two walls of protection against pathogens. Nat Rev Immunol 20, 229-238 (2020).

3. Schamel, W.W. \& Reth, M. Monomeric and oligomeric complexes of the B cell antigen receptor. Immunity 13, 5-14 (2000).

4. Hombach, J., Tsubata, T., Leclercq, L., Stappert, H. \& Reth, M. Molecular components of the Bcell antigen receptor complex of the IgM class. Nature 343, 760-762 (1990).

5. Tanaka, S. \& Baba, Y. B Cell Receptor Signaling. Adv Exp Med Biol 1254, 23-36 (2020).

6. Kwak, K., Akkaya, M. \& Pierce, S.K. B cell signaling in context. Nat Immunol 20, 963-969 (2019).

7. Tolar, P. \& Pierce, S.K. A conformation-induced oligomerization model for B cell receptor microclustering and signaling. Curr Top Microbiol Immunol 340, 155-169 (2010).

8. Yang, J. \& Reth, M. The dissociation activation model of B cell antigen receptor triggering. FEBS Letters 584, 4872-4877 (2010).

9. Metzger, H. Transmembrane signaling: the joy of aggregation. The Journal of Immunology 149, 1477-1487 (1992).

10. Weiss, A. \& Littman, D.R. Signal transduction by lymphocyte antigen receptors. Cell 76, $263-$ 274 (1994).

11. Harwood, N.E. \& Batista, F.D. Early events in B cell activation. Annu Rev Immunol 28, 185-210 (2010).

12. Pierce, S.K. \& Liu, W. The tipping points in the initiation of B cell signalling: how small changes make big differences. Nature Reviews Immunology 10, 767-777 (2010).

13. Lee, J., Sengupta, P., Brzostowski, J., Lippincott-Schwartz, J. \& Pierce, S.K. The nanoscale spatial organization of B-cell receptors on immunoglobulin M- and G-expressing human B-cells. Mol Biol Cell 28, 511-523 (2017).

14. Kim, Y.M., et al. Monovalent ligation of the B cell receptor induces receptor activation but fails to promote antigen presentation. Proc Natl Acad Sci U S A 103, 3327-3332 (2006).

Page 59 of 63 
1188

1189

1190

1191

1192

1193

1194

1195

1196

1197

1198

1199

1200

1201

1202

1203

1204

1205

1206

1207

1208

1209

1210

1211

1212

1213

1214

1215

1216

1217

1218

1219

1220

1221

1222

1223

1224

1225

1226

1227

1228

1229
15. Avalos, A.M., et al. Monovalent engagement of the BCR activates ovalbumin-specific transnuclear B cells. J Exp Med 211, 365-379 (2014).

16. Mukherjee, S., et al. Monovalent and multivalent ligation of the B cell receptor exhibit differential dependence upon Syk and Src family kinases. Sci Signal 6, ra1 (2013).

17. Yang, J. \& Reth, M. Oligomeric organization of the B-cell antigen receptor on resting cells. Nature 467, 465-469 (2010).

18. Davies, D.R., Padlan, E.A. \& Sheriff, S. Antibody-antigen complexes. Annu Rev Biochem 59, 439-473 (1990).

19. Minguet, S., Dopfer, E.P. \& Schamel, W.W. Low-valency, but not monovalent, antigens trigger the B-cell antigen receptor (BCR). Int Immunol 22, 205-212 (2010).

20. Tolar, P., Hanna, J., Krueger, P.D. \& Pierce, S.K. The constant region of the membrane immunoglobulin mediates B cell-receptor clustering and signaling in response to membrane antigens. Immunity 30, 44-55 (2009).

21. Zapadka, K.L., Becher, F.J., Gomes Dos Santos, A.L. \& Jackson, S.E. Factors affecting the physical stability (aggregation) of peptide therapeutics. Interface Focus 7, 20170030 (2017).

22. Jungmann, R., et al. Single-molecule kinetics and super-resolution microscopy by fluorescence imaging of transient binding on DNA origami. Nano Lett 10, 4756-4761 (2010).

23. Andersen, V.L., et al. A self-assembled, modular nucleic acid-based nanoscaffold for multivalent theranostic medicine. Theranostics 9, 2662-2677 (2019).

24. Shih, T.A., Roederer, M. \& Nussenzweig, M.C. Role of antigen receptor affinity in T cellindependent antibody responses in vivo. Nat Immunol 3, 399-406 (2002).

25. Pleiner, T., Bates, M. \& Görlich, D. A toolbox of anti-mouse and anti-rabbit IgG secondary nanobodies. Journal of Cell Biology 217, 1143-1154 (2017).

26. Ester, M., Kriegel, H.-P., Sander, J. \& Xu, X. A Density-Based Algorithm for Discovering Clusters in Large Spatial Databases with Noise. Association for the Advancement of Artificial Intelligence 226-231 (1996).

27. Jungmann, R., et al. Quantitative super-resolution imaging with qPAINT. Nat Methods 13, 439442 (2016). 
28. Monroe, J.G. \& Cambier, J.C. Sorting of B lymphoblasts based upon cell diameter provides cell populations enriched in different stages of cell cycle. J Immunol Methods 63, 45-56 (1983).

29. Reth, M., Hammerling, G.J. \& Rajewsky, K. Analysis of the repertoire of anti-NP antibodies in C57BL/6 mice by cell fusion. I. Characterization of antibody families in the primary and hyperimmune response. Eur J Immunol 8, 393-400 (1978).

30. Sonoda, E., et al. B cell development under the condition of allelic inclusion. Immunity 6, 225233 (1997).

31. Pedersen, M.E., Haegebaert, R.M.S., Ostergaard, J. \& Jensen, H. Size-based characterization of adalimumab and TNF-alpha interactions using flow induced dispersion analysis: assessment of avidity-stabilized multiple bound species. Sci Rep-Uk 11, (2021).

32. Pedersen, M.E., Ostergaard, J. \& Jensen, H. Flow-Induced Dispersion Analysis (FIDA) for Protein Quantification and Characterization. Methods Mol Biol 1972, 109-123 (2019).

33. Jensen, H. \& Ostergaard, J. Flow induced dispersion analysis quantifies noncovalent interactions in nanoliter samples. Journal of the American Chemical Society 132, 4070-4071 (2010).

34. Liu, W., Meckel, T., Tolar, P., Sohn, H.W. \& Pierce, S.K. Antigen affinity discrimination is an intrinsic function of the B cell receptor. J Exp Med 207, 1095-1111 (2010).

35. Svitel, J., Balbo, A., Mariuzza, R.A., Gonzales, N.R. \& Schuck, P. Combined affinity and rate constant distributions of ligand populations from experimental surface binding kinetics and equilibria. Biophys J 84, 4062-4077 (2003).

36. Gorshkova, II, Svitel, J., Razjouyan, F. \& Schuck, P. Bayesian analysis of heterogeneity in the distribution of binding properties of immobilized surface sites. Langmuir 24, 11577-11586 (2008).

37. Vorup-Jensen, T. On the roles of polyvalent binding in immune recognition: perspectives in the nanoscience of immunology and the immune response to nanomedicines. Adv Drug Deliv Rev 64, 1759-1781 (2012).

38. Hauser, A.E., et al. Definition of germinal-center B cell migration in vivo reveals predominant intrazonal circulation patterns. Immunity 26, 655-667 (2007).

39. Baumgart, F., Arnold, A.M., Rossboth, B.K., Brameshuber, M. \& Schutz, G.J. What we talk about when we talk about nanoclusters. Methods Appl Fluoresc 7, 013001 (2018).

40. Annibale, P., Vanni, S., Scarselli, M., Rothlisberger, U. \& Radenovic, A. Identification of clustering artifacts in photoactivated localization microscopy. Nat Methods 8, 527-528 (2011). 
1273

1274

1275

1276

1277

1278

1279

1280

1281

1282

1283

1284

1285

1286

1287

1288

1289

1290

1291

1292

1293

1294

1295

1296

1297

1298

1299

1300

1301

1302

1303

1304

1305

1306

1307

1308

1309

1310

1311

1312

1313

1314

1315

41. Gasparrini, F., et al. Nanoscale organization and dynamics of the siglec CD22 cooperate with the cytoskeleton in restraining BCR signalling. EMBO J 35, 258-280 (2016).

42. Mattila, P.K., et al. The actin and tetraspanin networks organize receptor nanoclusters to regulate B cell receptor-mediated signaling. Immunity 38, 461-474 (2013).

43. Maity, P.C., et al. B cell antigen receptors of the IgM and IgD classes are clustered in different protein islands that are altered during B cell activation. Sci Signal 8, ra93 (2015).

44. Garcia-Parajo, M.F., Cambi, A., Torreno-Pina, J.A., Thompson, N. \& Jacobson, K.

Nanoclustering as a dominant feature of plasma membrane organization. $J$ Cell Sci 127, 49955005 (2014).

45. Gold, M.R. \& Reth, M.G. Antigen Receptor Function in the Context of the Nanoscale Organization of the B Cell Membrane. Annu Rev Immunol 37, 97-123 (2019).

46. Brameshuber, M., et al. Monomeric TCRs drive T cell antigen recognition. Nat Immunol 19, 487496 (2018).

47. Rossboth, B., et al. TCRs are randomly distributed on the plasma membrane of resting antigenexperienced T cells. Nat Immunol 19, 821-827 (2018).

48. Volkmann, C., et al. Molecular requirements of the B-cell antigen receptor for sensing monovalent antigens. EMBO J 35, 2371-2381 (2016).

49. Gjelstrup, L.C., et al. The role of nanometer-scaled ligand patterns in polyvalent binding by large mannan-binding lectin oligomers. J Immunol 188, 1292-1306 (2012).

50. Kitov, P.I. \& Bundle, D.R. On the nature of the multivalency effect: a thermodynamic model. $J$ Am Chem Soc 125, 16271-16284 (2003).

51. Germain, R.N. T-cell signaling: the importance of receptor clustering. Curr Biol 7, R640-644 (1997).

52. Davis, S.J. \& van der Merwe, P.A. The structure and ligand interactions of CD2: implications for T-cell function. Immunol Today 17, 177-187 (1996).

53. Radaev, S., et al. Structural and functional studies of Igalphabeta and its assembly with the B cell antigen receptor. Structure 18, 934-943 (2010).

54. Shen, Z., et al. Conformational change within the extracellular domain of B cell receptor in B cell activation upon antigen binding. Elife 8, (2019). 
55. Jensen, M.R., et al. Structural basis for simvastatin competitive antagonism of complement receptor 3. Journal of Biological Chemistry 291, 16963-16976 (2016).

56. Juul-Madsen, K., et al. Size-Selective Phagocytic Clearance of Fibrillar $\alpha$-Synuclein through Conformational Activation of Complement Receptor 4. The Journal of Immunology 204, 13451361 (2020).

57. Zhao, H., Gorshkova, II, Fu, G.L. \& Schuck, P. A comparison of binding surfaces for SPR biosensing using an antibody-antigen system and affinity distribution analysis. Methods 59, 328335 (2013).

58. Jarmoskaite, I., AlSadhan, I., Vaidyanathan, P.P. \& Herschlag, D. How to measure and evaluate binding affinities. Elife 9, (2020).

59. Bailey, S. \& Macardle, P.J. A flow cytometric comparison of Indo-1 to fluo-3 and Fura Red excited with low power lasers for detecting $\mathrm{Ca}(2+)$ flux. $J$ Immunol Methods 311, 220-225 (2006).

60. Hansen, S.B., Laursen, N.S., Andersen, G.R. \& Andersen, K.R. Introducing site-specific cysteines into nanobodies for mercury labelling allows de novo phasing of their crystal structures. Acta Crystallographica Section D: Structural Biology 73, 804-813 (2017).

61. Schnitzbauer, J., Strauss, M.T., Schlichthaerle, T., Schueder, F. \& Jungmann, R. Super-resolution microscopy with DNA-PAINT. Nat Protoc 12, 1198-1228 (2017).

62. Endesfelder, U., Malkusch, S., Fricke, F. \& Heilemann, M. A simple method to estimate the average localization precision of a single-molecule localization microscopy experiment. Histochem Cell Biol 141, 629-638 (2014).

63. Strauss, S. \& Jungmann, R. Up to 100-fold speed-up and multiplexing in optimized DNAPAINT. Nat Methods 17, 789-791 (2020).

64. Fischer, L.S., et al. Quantitative single-protein imaging reveals molecular complex formation of integrin, talin, and kindlin during cell adhesion. Nat Commun 12, 919 (2021).

65. Wade, O.K., et al. 124-Color Super-resolution Imaging by Engineering DNA-PAINT Blinking Kinetics. Nano Lett 19, 2641-2646 (2019). 